\title{
Paracomplex Paracontact Pseudo-Riemannian Submersions
}

\author{
S. S. Shukla and Uma Shankar Verma \\ Department of Mathematics, University of Allahabad, Allahabad 211002, India \\ Correspondence should be addressed to Uma Shankar Verma; umashankarverma7@gmail.com
}

Received 25 February 2014; Accepted 7 April 2014; Published 7 May 2014

Academic Editor: Bennett Palmer

Copyright (C) 2014 S. S. Shukla and U. S. Verma. This is an open access article distributed under the Creative Commons Attribution License, which permits unrestricted use, distribution, and reproduction in any medium, provided the original work is properly cited.

\begin{abstract}
We introduce the notion of paracomplex paracontact pseudo-Riemannian submersions from almost para-Hermitian manifolds onto almost paracontact metric manifolds. We discuss the transference of structures on total manifolds and base manifolds and provide some examples. We also obtain the integrability condition of horizontal distribution and investigate curvature properties under such submersions.
\end{abstract}

\section{Introduction}

The theory of Riemannian submersion was introduced by O’Neill [1,2] and Gray [3]. It is known that the applications of such Riemannian submersion are extensively used in KaluzaKlein theories [4, 5], Yang-Mill equations [6, 7], the theory of robotics [8], and supergravity and superstring theories [5,9].

There is detailed literature on the Riemannian submersion with suitable smooth surjective map followed by different conditions applied to total space and on the fibres of surjective map. The Riemannian submersions between almost Hermitian manifolds have been studied by Watson [10]. The Riemannian submersions between almost contact manifolds were studied by Chinea [11]. He also concluded that if $\bar{M}$ is an almost Hermitian manifold with structure $(\bar{J}, \bar{g})$ and $M$ is an almost contact metric manifold with structure $(\phi, \xi, \eta, g)$, then there does not exist a Riemannian submersion $f: \bar{M} \rightarrow$ $M$ which commutes with the structures on $\bar{M}$ and $M$; that is, we cannot have the condition $f_{*} \circ \bar{J}=\phi \circ f_{*}$. Chinea also defined the Riemannian submersion between almost complex manifolds and almost contact manifolds and studied some properties and interrelations between them [12]. In [13], Gündüzalp and Sahin gave the concept of paracontact paracomplex semi-Riemannian submersion between almost paracontact metric manifolds and almost para-Hermitian manifolds submersion giving an example and studied some geometric properties of such submersions.
An almost paracontact structure on a differentiable manifold was introduced by Sato [14], which is an analogue of an almost contact structure and is closely related to almost product structure. An almost contact manifold is always odd dimensional but an almost paracontact manifold could be even dimensional as well.

The paracomplex geometry has been studied since the first papers by Rashevskij [15], Libermann [16], and Patterson [17] until now, from several different points of view. The subject has applications to several topics such as negatively curved manifolds, mechanics, elliptic geometry, and pseudoRiemannian space forms. Paracomplex and paracontact geometries are topics with many analogies and also with differences with complex and contact geometries.

This motivated us to study the pseudo-Riemannian submersion between pseudo-Riemannian manifolds equipped with paracomplex and paracontact structures.

In this paper, we give the notion of paracomplex paracontact pseudo-Riemannian submersion between almost paracomplex manifolds and almost paracontact pseudometric manifolds giving some examples and study the geometric properties and interrelations under such submersions.

The composition of the paper is as follows. In Section 2, we collect some basic definitions, formulas, and results on almost paracomplex manifolds, almost paracontact pseudometric manifolds, and pseudo-Riemannian submersion. 
In Section 3, we define paracomplex paracontact pseudoRiemannian submersion giving some relevant examples and investigate transference of structures on the total manifolds and base manifolds under such submersions. In Section 4, curvature relations between total manifolds, base manifolds, and fibres are studied.

\section{Preliminaries}

2.1. Almost Paracontact Manifolds. Let $M$ be a $(2 n+1)$ dimensional Riemannian manifold, $\phi$ a (1,1)-type tensor field, $\xi$ a vector field, called characteristic vector field, and $\eta$ a 1form on $M$. Then, $(\phi, \xi, \eta)$ is called an almost paracontact structure on $M$ if

$$
\phi^{2} X=X-\eta(X) \xi ; \quad \eta(\xi)=1,
$$

and the tensor field $\phi$ induces an almost paracomplex structure on the distribution $\mathscr{D}=\operatorname{ker}(\eta)[18,19]$.

$M$ is said to be an almost paracontact manifold, if it is equipped with an almost paracontact structure. Again, $M$ is called an almost paracontact pseudometric manifold if it is endowed with a pseudo-Riemannian metric $g$ of signature $\underbrace{(-,-,-, \ldots,-}_{(n \text {-times })}, \underbrace{+,+,+, \ldots,+)}_{(n+1) \text {-times })}$ such that

$$
g(\phi X, \phi Y)=g(X, Y)-\varepsilon \eta(X) \eta(Y), \quad \forall X, Y \in \Gamma(T M),
$$

where $\varepsilon=1$ or -1 according to the characteristic vector field $\xi$ is spacelike or timelike. It follows that

$$
\begin{gathered}
g(\xi, \xi)=\varepsilon, \\
g(\xi, X)=\varepsilon \eta(X), \\
g(X, \phi Y)=g(\phi X, Y), \quad \forall X, Y \in \Gamma(T M) .
\end{gathered}
$$

In particular, if index $(g)=1$, then the manifold $\left(M^{2 n+1}, \phi, \xi, \eta, g, \varepsilon\right)$ is called a Lorentzian almost paracontact manifold.

If the metric $g$ is positive definite, then the manifold $\left(M^{2 n+1}, \phi, \xi, \eta, g\right)$ is the usual almost paracontact metric manifold [14].

The fundamental 2-form $\Phi$ on $M$ is defined by

$$
\Phi(X, Y)=g(X, \phi Y) \text {. }
$$

Let $M^{2 n+1}$ be an almost paracontact manifold with the structure $(\phi, \xi, \eta)$. An almost paracomplex structure $J$ on $M^{2 n+1} \times \mathbb{R}^{1}$ is defined by

$$
J\left(X, f \frac{d}{d t}\right)=\left(\phi X+f \xi, \eta(X) \frac{d}{d t}\right),
$$

where $X$ is tangent to $M^{2 n+1}, t$ is the coordinate on $\mathbb{R}^{1}$, and $f$ is a smooth function on $M^{2 n+1}$.

An almost paracontact structure $(\phi, \xi, \eta)$ is said to be normal, if the Nijenhuis tensor $N_{J}$ of almost paracomplex structure $J$ defined as

$$
\begin{aligned}
N_{J}(X, Y)= & {[J, J](X, Y)=[J X, J Y]+J^{2}[X, Y] } \\
& -J[J X, Y]-J[X, J Y],
\end{aligned}
$$

for any vector fields $X, Y \in \Gamma(T M)$, vanishes.
20]

If $X$ and $Y$ are vector fields on $M^{2 n+1}$, then we have [18-

$$
\begin{gathered}
N_{J}((X, 0),(Y, 0)) \\
=\left(N_{\phi}(X, Y)-2 d \eta(X, Y) \xi,\right. \\
\left.\left\{\left(\mathscr{L}_{\phi X} \eta\right) Y-\left(\mathscr{L}_{\phi Y} \eta\right) X\right\} \frac{d}{d t}\right), \\
N_{J}\left((X, 0),\left(0, \frac{d}{d t}\right)\right)=-\left(\left(\mathscr{L}_{\xi} \phi\right) X,\left(\left(\mathscr{L}_{\xi} \eta\right) X\right) \frac{d}{d t}\right),
\end{gathered}
$$

where $N_{\phi}$ is Nijenhuis tensor of $\phi, \mathscr{L}_{X}$ is Lie derivative with respect to a vector field $X$, and $N^{(1)}, N^{(2)}, N^{(3)}$, and $N^{(4)}$ are defined as

$$
\begin{gathered}
N_{\phi}(X, Y) \\
=[\phi, \phi](X, Y) \\
=[\phi X, \phi Y]+\phi^{2}[X, Y]-\phi[\phi X, Y]-\phi[X, \phi Y], \\
N^{(1)}(X, Y)=N_{\phi}(X, Y)-2 d \eta(X, Y) \xi, \\
N^{(2)}(X, Y)=\left(\mathscr{L}_{\phi X} \eta\right) Y-\left(\mathscr{L}_{\phi Y} \eta\right) X, \\
N^{(3)}(X)=\left(\mathscr{L}_{\xi} \phi\right) X, \\
N^{(4)}(X)=\left(\mathscr{L}_{\xi} \eta\right) X .
\end{gathered}
$$

The almost paracontact structure $(\phi, \xi, \eta)$ is normal if and only if the four tensors $N^{(1)}, N^{(2)}, N^{(3)}$, and $N^{(4)}$ vanish.

For an almost paracontact structure $(\phi, \xi, \eta)$, vanishing of $N^{(1)}$ implies the vanishing of $N^{(2)}, N^{(3)}$, and $N^{(4)}$. Moreover, $N^{(2)}$ vanishes if and only if $\xi$ is a killing vector field.

An almost paracontact pseudometric manifold $\left(M^{2 n+1}, \phi, \xi, \eta, g, \varepsilon\right)$ is called

(i) normal, if $N_{\phi}-2 d \eta \otimes \xi=0$,

(ii) paracontact, if $\Phi=d \eta$,

(iii) $K$-paracontact, if $M$ is paracontact and $\xi$ is killing,

(iv) paracosymplectic, if $\nabla \Phi=0$, which implies $\nabla \eta=0$, where $\nabla$ is the Levi-Civita connection on $M$,

(v) almost paracosymplectic, if $d \eta=0$ and $d \Phi=0$,

(vi) weakly paracosymplectic, if $M$ is almost paracosymplectic and $[R(X, Y), \phi]=R(X, Y) \phi-\phi R(X, Y)=0$, where $R$ is Riemannian curvature tensor,

(vii) para-Sasakian, if $\Phi=d \eta$ and $M$ is normal,

(viii) quasi-para-Sasakian, if $d \phi=0$ and $M$ is normal.

2.2. Almost Paracomplex Manifolds. A (1,1)-type tensor field $J$ on $2 m$-dimensional smooth manifold $M$ is said to be an almost paracomplex structure if $J^{2}=I$ and $\left(M^{2 m}, J\right)$ is called almost paracomplex manifold. 
An almost paracomplex manifold $(M, J)$ is such that the two eigenbundles $T^{+} M$ and $T^{-} M$ corresponding to respective eigenvalues +1 and -1 of $J$ have the same rank $[21,22]$.

An almost para-Hermitian manifold $(M, J, g)$ is a smooth manifold endowed with an almost paracomplex structure $J$ and a pseudo-Riemannian metric $g$ such that

$$
g(J X, J Y)=-g(X, Y), \quad \forall X, Y \in \Gamma(T M) .
$$

Here, the metric $g$ is neutral; that is, $g$ has signature $(m, m)$.

The fundamental 2-form of the almost para-Hermitian manifold is defined by

$$
F(X, Y)=g(X, J Y) .
$$

We have the following properties $[21,22]$ :

$$
\begin{gathered}
g(J X, Y)=-g(X, J Y), \\
F(X, Y)=-F(Y, X), \\
F(J X, J Y)=-F(X, Y),
\end{gathered}
$$

$3 d F(X, Y, Z)$

$$
\begin{gathered}
=X(F(Y, Z))-Y(F(X, Z))+Z(F(X, Y)) \\
-F([X, Y], Z)+F([X, Z], Y)-F([Y, Z], X), \\
\left(\nabla_{X} F\right)(Y, Z)=g\left(Y,\left(\nabla_{X} J\right) Z\right)=-g\left(Z,\left(\nabla_{X} J\right) Y\right), \\
3 d F(X, Y, Z)=\left(\nabla_{X} F\right)(Y, Z)+\left(\nabla_{Y} F\right)(Z, X) \\
+\left(\nabla_{Z} F\right)(X, Y),
\end{gathered}
$$

the co-differential, $(\delta F)(X)=\sum_{i=1}^{2 m} \varepsilon_{i}\left(\nabla_{e_{i}} F\right)\left(e_{i}, X\right)$.

An almost para-Hermitian manifold is called

(i) para-Hermitian, if $N_{J}=0$; equivalently, $\left(\nabla_{J X} J\right) J Y+$ $\left(\nabla_{X} J\right) Y=0$

(ii) para-Kähler, if, for any $X \in \Gamma(T M), \nabla_{X} J=0$; that is, $\nabla J=0$,

(iii) almost para-Kähler, if $d F=0$,

(iv) nearly para-Kähler, if $\left(\nabla_{X} J\right) X=0$,

(v) almost semi-para-Kähler, if $\delta F=0$,

(vi) semi-para-Kähler, if $\delta F=0$ and $N_{J}=0$.

2.3. Pseudo-Riemannian Submersion. Let $\left(\bar{M}^{m}, \bar{g}\right)$ and $\left(M^{n}, g\right)$ be two connected pseudo-Riemannian manifolds of indices $\bar{s}(0 \leq \bar{s} \leq m)$ and $s(0 \leq s \leq n)$, respectively, with $\bar{s} \geq s$.

A pseudo-Riemannian submersion is a smooth map $f$ : $\bar{M}^{m} \rightarrow M^{n}$, which is onto and satisfies the following conditions $[2,3,23,24]$.

(i) The derivative map $f_{*_{p}}: T_{p} \bar{M} \rightarrow T_{f(p)} M$ is surjective at each point $p \in \bar{M}$. (ii) The fibres $f^{-1}(q)$ of $f$ over $q \in M$ are either pseudo-Riemannian submanifolds of $\bar{M}$ of dimension $(m-n)$ and index $v$ or the degenerate submanifolds of $\bar{M}$ of dimension $(m-n)$ and index $v$ with degenerate metric $\bar{g}_{\left.\right|_{f^{-1}(q)}}$ of type $(\underbrace{0,0,0, \ldots, 0}_{\mu \text {-times }}, \underbrace{-,-,-, \ldots,-}_{\nu \text {-times }}, \underbrace{+,+,+, \ldots,+}_{(m-n-\mu-\nu \text {-times })}), \quad$ where $\mu=\operatorname{dim}\left(\mathscr{V}_{p} \cap \mathscr{H}_{p}\right)$ and $v=\bar{s}-s=$ index of $\bar{g}_{\left.\right|_{f^{-1}(q)}}$.

(iii) $f_{*}$ preserves the length of horizontal vectors.

We denote the vertical and horizontal projections of a vector field $E$ on $\bar{M}$ by $E^{v}$ (or by $v E$ ) and $E^{h}$ (or by $h E$ ), respectively. A horizontal vector field $\bar{X}$ on $\bar{M}$ is said to be basic if $\bar{X}$ is $f$-related to a vector field $X$ on $M$. Thus, every vector field $X$ on $M$ has a unique horizontal lift $\bar{X}$ on $\bar{M}$.

Lemma 1 (see $[1,23])$. If $f: \bar{M} \rightarrow M$ is a pseudoRiemannian submersion and $\bar{X}, \bar{Y}$ are basic vector fields on $\bar{M}$ that are $f$-related to the vector fields $X, Y$ on $M$, respectively, then one has the following properties:

(i) $\bar{g}(\bar{X}, \bar{Y})=g(X, Y) \circ f$,

(ii) $h[\bar{X}, \bar{Y}]$ is a vector field and $h[\bar{X}, \bar{Y}]=[X, Y] \circ f$,

(iii) $h(\bar{\nabla} \bar{X} \bar{Y})$ is a basic vector field $f$-related to $\nabla_{X} Y$, where $\bar{\nabla}$ and $\nabla$ are the Levi-Civita connections on $\bar{M}$ and $M$, respectively,

(iv) $[E, U] \in \mathscr{V}$, for any vector field $U \in \mathscr{V}$ and for any vector field $E \in \Gamma(T \bar{M})$.

A pseudo-Riemannian submersion $f: \bar{M} \rightarrow M$ determines tensor fields $\mathscr{T}$ and $\mathscr{A}$ of type $(1,2)$ on $\bar{M}$ defined by formulas $[1,2,23]$

$$
\begin{gathered}
\mathscr{T}(E, F)=\mathscr{T}_{E} F=h\left(\bar{\nabla}_{v E} v F\right)+v\left(\bar{\nabla}_{v E} h F\right), \\
\mathscr{A}(E, F)=\mathscr{A}_{E} F=v\left(\bar{\nabla}_{h E} h F\right)+h\left(\bar{\nabla}_{h E} v F\right),
\end{gathered}
$$

for any $E, F \in \Gamma(T \bar{M})$.

Let $\bar{X}, \bar{Y}$ be horizontal vector fields and let $U, V$ be vertical vector fields on $\bar{M}$. Then, one has

$$
\begin{gathered}
\mathscr{T}_{U} \bar{X}=v\left(\bar{\nabla}_{U} \bar{X}\right), \quad \mathscr{T}_{U} V=h\left(\bar{\nabla}_{U} V\right), \\
\bar{\nabla}_{U} \bar{X}=\mathscr{T}_{U} \bar{X}+h\left(\bar{\nabla}_{U} \bar{X}\right), \\
\mathscr{T}_{\bar{X}} F=0, \quad \mathscr{T}_{E} F=\mathscr{T}_{v E} F, \\
\bar{\nabla}_{U} V=\mathscr{T}_{U} V+v\left(\bar{\nabla}_{U} V\right), \\
\mathscr{A}_{\bar{X}} \bar{Y}=v\left(\bar{\nabla}_{\bar{X}} \bar{Y}\right), \quad \mathscr{A}_{\bar{X}} U=h\left(\bar{\nabla}_{\bar{X}} U\right), \\
\bar{\nabla}_{\bar{X}} U=\mathscr{A}_{\bar{X}} U+v\left(\bar{\nabla}_{\bar{X}} U\right), \\
\mathscr{A}_{U} F=0, \quad \mathscr{A}_{E} F=\mathscr{A}_{h E} F, \\
\bar{\nabla}_{\bar{X}} \bar{Y}=\mathscr{A}_{\bar{X}} \bar{Y}+h\left(\nabla_{\bar{X}} \bar{Y}\right),
\end{gathered}
$$




$$
\begin{gathered}
h\left(\bar{\nabla}_{U} \bar{X}\right)=h\left(\bar{\nabla}_{\bar{X}} U\right)=\mathscr{A}_{\bar{X}} U, \\
\mathscr{A}_{\bar{X}} \bar{Y}=\frac{1}{2} v[\bar{X}, \bar{Y}], \\
\mathscr{A}_{\bar{X}} \bar{Y}=-\mathscr{A}_{\bar{Y}} \bar{X} \\
\mathscr{T}_{U} V=\mathscr{T}_{V} U,
\end{gathered}
$$

for all $E, F \in \Gamma(T \bar{M})$.

Moreover, $\mathscr{T}_{U} V$ coincides with second fundamental form of the submersion of the fibre submanifolds. The distribution $\mathscr{H}$ is completely integrable. In view of (37) and (38), $\mathscr{A}$ is alternating on the horizontal distribution and $\mathscr{T}$ is symmetric on the vertical distribution.

\section{Paracomplex Paracontact Pseudo- Riemannian Submersions}

In this section, we introduce the notion of pseudoRiemannian submersion from almost paracomplex manifolds onto almost paracontact pseudometric manifolds, illustrate examples, and study the transference of structures on total manifolds and base manifolds.

Definition 2. Let $\left(\bar{M}^{2 m}, \bar{J}, \bar{g}\right)$ be an almost para-Hermitian manifold and let $\left(M^{2 n+1}, \phi, \xi, \eta, g\right)$ be an almost paracontact pseudometric manifold.

A pseudo-Riemannian submersion $f: \bar{M} \rightarrow M$ is called paracomplex paracontact pseudo-Riemannian submersion if there exists a 1 -form $\bar{\eta}$ on $\bar{M}$ such that

$$
f_{*} \circ \bar{J}=\phi \circ f_{*}+\bar{\eta} \otimes \xi .
$$

Since, for each $p \in \bar{M}, f_{*_{p}}$ is a linear isometry between horizontal spaces $\mathscr{H}_{p}$ and tangent spaces $T_{f(p)} M$, there exists an induced almost paracontact structure $\left(\bar{\phi}^{h}, \bar{\eta}^{h}, \bar{\xi}^{h}, \bar{g}\right)$ on $(2 n+1)$-dimensional horizontal distribution $\mathscr{H}$ such that $\bar{\phi}_{\left.\right|_{\bar{D}^{h}}}^{h}$ behave just like the fundamental collineation of almost paracomplex structure $\bar{J}$ on ker $\bar{\eta}^{h}=\overline{\mathscr{D}}^{h}$ and $\bar{\phi}^{h}: \overline{\mathscr{D}}^{h} \rightarrow \overline{\mathscr{D}}^{h}$ is an endomorphism such that $\bar{\phi}^{h}=\bar{J}_{\left.\right|_{\text {ker } \bar{\eta}^{h}}}$ and the rank of $\bar{\phi}^{h}=2 n$, where $\operatorname{dim}\left(\overline{\mathscr{D}}^{h}\right)=2 n$.

It follows that, for any $\bar{X}^{h} \in \overline{\mathscr{D}}^{h}, \bar{\eta}^{h}\left(\bar{X}^{h}\right)=0$, which implies that $\bar{J}_{\left.\right|_{\bar{D}^{h}}}^{2}\left(\bar{X}^{h}\right)=\left(\bar{\phi}^{h}\right)^{2}\left(\bar{X}^{h}\right)=\bar{X}^{h}$, for any $\bar{X}^{h} \in \overline{\mathscr{D}}^{h}$ and $\mathscr{H}=\overline{\mathscr{D}}^{h} \oplus\left\{\bar{\xi}^{h}\right\}[18]$.

Definition 3 (see [25]). A pseudo-Riemannian submersion $f: \bar{M} \rightarrow M$ is called semi- $\bar{J}$-invariant submersion, if there is a distribution $\overline{\mathscr{D}}_{1} \subseteq \operatorname{ker} f_{*}$ such that

$$
\begin{gathered}
\operatorname{ker} f_{*}=\overline{\mathscr{D}}_{1} \oplus \overline{\mathscr{D}}_{2}, \\
\bar{J}\left(\overline{\mathscr{D}}_{1}\right)=\overline{\mathscr{D}}_{1}, \quad \bar{J}\left(\overline{\mathscr{D}}_{2}\right) \subseteq\left(\operatorname{ker} f_{*}\right)^{\perp},
\end{gathered}
$$

where $\overline{\mathscr{D}}_{2}$ is orthogonal complementary to $\overline{\mathscr{D}}_{1}$ in ker $f_{*}$.
Proposition 4. Let $f: \bar{M}^{2 m} \rightarrow M^{2 n+1}$ be a paracomplex paracontact pseudo-Riemannian submersion and let the fibres of $f$ be pseudo-Riemannian submanifolds of $\bar{M}$. Then, the fibres $f^{-1}(q), q \in M$, are semi-J-invariant submanifolds of $\bar{M}$ of dimension $(2 m-2 n-1)$.

Proof. Let $\bar{U} \in \mathscr{V}$. Then

$$
\begin{gathered}
f_{*}(\overline{J U})=\phi\left(f_{*}(\bar{U})\right)+\bar{\eta}(\bar{U}) \xi \\
\Longrightarrow f_{*}\left\{\bar{J}(\bar{U})-\bar{\eta}(\bar{U}) \bar{\xi}^{h}\right\}=0
\end{gathered}
$$

where $f_{*} \bar{\xi}^{h}=\xi$.

Thus, we have

$$
\bar{J}(\bar{U})-\bar{\eta}(\bar{U}) \bar{\xi}^{h}=\bar{\phi}(\bar{U}), \quad \text { for some } \bar{\phi}(\bar{U}) \in \mathscr{V} \text {. }
$$

By (19), we get $\bar{g}\left(\bar{\xi}^{h}, \bar{J}\left(\bar{\xi}^{h}\right)\right)=0=g\left(\xi, f_{*}\left(\bar{J}\left(\bar{\xi}^{h}\right)\right)\right)=0$.

As $g$ is nondegenerate on $M$, we have

$$
f_{*}\left(\bar{J}\left(\bar{\xi}^{h}\right)\right)=0, \quad \text { that is } \bar{J}\left(\bar{\xi}^{h}\right) \in \mathscr{V} \text {. }
$$

Taking $\bar{U}=\bar{J} \bar{\xi}^{h}$ in (43), we obtain

$$
\bar{\xi}^{h}-\bar{\eta}\left(\bar{J} \bar{\xi}^{h}\right) \bar{\xi}^{h}=\bar{\phi}\left(\bar{J} \bar{\xi}^{h}\right) .
$$

Since fibre $f^{-1}(q)$ is an odd dimensional submanifold, there exists an associated 1-form $\bar{\eta}^{v}$ which is restriction of $\bar{\eta}$ on fibre submanifold $f^{-1}(q), q \in M$, and a characteristic vector field $\bar{\xi}^{v}=\bar{J} \bar{\xi}^{h}$ such that $\bar{\phi}\left(\bar{\xi}^{v}\right)=0$. So, we have $\bar{\eta}^{v}\left(\bar{\xi}^{v}\right)=1$.

Let us put ker $\bar{\eta}^{v}=\overline{\mathscr{D}}_{1}$ and $\overline{\mathscr{D}}_{2}=\left\{\bar{\xi}^{v}\right\}$.

Then, ker $f_{*}=\overline{\mathscr{D}}_{1} \oplus \overline{\mathscr{D}}_{2}$ and $\bar{J}\left(\overline{\mathscr{D}}_{1}\right)=\mathscr{D}_{1}, \bar{J}\left(\overline{\mathscr{D}}_{2}\right)=$ $\bar{J}\left\{\bar{\xi}^{v}\right\}=\left\{\bar{\xi}^{h}\right\} \subseteq\left(\operatorname{ker} f_{*}\right)^{\perp}$.

Hence, the fibres $f^{-1}(q)$ are semi- $\bar{J}$-invariant submanifolds of $\bar{M}$.

Corollary 5. Let $f: \bar{M}^{2 m} \rightarrow M^{2 n+1}$ be a paracomplex paracontact pseudo-Riemannian submersion and let the fibres of $f$ be pseudo-Riemannian submanifolds of $\bar{M}$. Then, the fibres $f^{-1}(q)$ are almost paracontact pseudometric manifolds with almost paracontact pseudo-Riemannian structures $\left(\bar{\phi}^{v}, \bar{\xi}^{v}, \bar{\eta}^{v}, \bar{g}^{v}\right), q \in M$, where $\bar{\xi}^{v}=\bar{J}\left(\bar{\xi}^{h}\right), \bar{\eta}^{v}=\bar{\eta}_{\left.\right|_{\mathscr{V}}}$, and $\bar{g}^{v}=\bar{g}$.

Proof. Since $f^{-1}(q)$ are semi- $\bar{J}$-invariant submanifolds of $\bar{M}$ of odd dimension $2 r+1=2 m-2 n-1$, (39) implies

$$
\bar{J}(\bar{U})=\bar{\phi}^{v} \bar{U}+\bar{\eta}^{v}(\bar{U}) \bar{\xi}^{h}
$$

for any $\bar{U} \in \mathscr{V}$. 

get

On operating $\bar{J}$ on both sides of the above equation, we

$$
\bar{U}=\bar{\phi}^{v}\left(\bar{\phi}^{v}(\bar{U})\right)+\bar{\eta}^{v}\left(\bar{\phi}^{v}(\bar{U})\right) \bar{\xi}^{h}+\bar{\eta}^{v}(\bar{U}) \bar{\xi}^{v}
$$

where $\bar{J}\left(\bar{\xi}^{h}\right)=\bar{\xi}^{v}$.

Equating horizontal and vertical components, we have

$$
\begin{gathered}
\bar{U}=\bar{\phi}^{v}\left(\bar{\phi}^{v}(\bar{U})\right)+\bar{\eta}^{v}(\bar{U}) \bar{\xi}^{v}, \quad \bar{\eta}^{v} \circ \bar{\phi}^{v}(\bar{U})=0, \\
\Longrightarrow\left(\bar{\phi}^{v}\right)^{2}(\bar{U})=\bar{U}-\bar{\eta}^{v}(\bar{U}) \bar{\xi}^{v} ; \quad \bar{\eta}^{v} \circ \bar{\phi}^{v}=0 ; \\
\bar{\phi}^{v}\left(\bar{\xi}^{v}\right)=0, \quad \bar{\eta}^{v}\left(\bar{\xi}^{v}\right)=1 .
\end{gathered}
$$

Hence, $\left(\bar{\phi}^{v}, \bar{\xi}^{v}, \bar{\eta}^{v}, \bar{g}^{v}\right)$ is almost paracontact pseudometric structure on the fibre $f^{-1}(q), q \in M$.

Proposition 6. Let $f: \bar{M}^{2 m} \rightarrow M^{2 n+1}$ be a paracomplex paracontact pseudo-Riemannian submersion and let the fibres of $f$ be pseudo-Riemannian submanifolds of $\bar{M}$. Let $\bar{X}, \bar{Y}$ be basic vector fields $f$-related to $X, Y$, respectively. Let $\bar{\eta}$ and $\eta$ be 1-forms on the total manifold $\bar{M}$ and the base manifold $M$, respectively. Then, one has the following.

(i) The characteristic vector field $\bar{J} \bar{\xi}^{h}$ is a vertical vector field.

(ii) $f_{*}^{*} \eta=\bar{\eta}^{h}$, where $f_{*}^{*} \eta$ is pullback of $\eta$ through $f_{*}$.

(iii) $\bar{\eta}^{h}(\bar{U})=0$, for any vertical vector field $\bar{U}$.

(iv) $\bar{\eta}^{v}(\bar{X})=0$, for any horizontal vector field $\bar{X}$.

Remark 7. Results (ii) and (iv) are analogue version of results (i) and (iii) of Proposition 4 of [13].

Proof. (i) By Corollary 5, $\left(\bar{\phi}^{v}, \bar{\xi}^{v}, \bar{\eta}^{v}, \bar{g}^{v}\right)$ is almost paracontact pseudometric structure on $f^{-1}(q)$. We have

$$
\begin{aligned}
0 & =\bar{g}\left(\bar{\xi}^{h}, \bar{J}^{h}\right)=g\left(f_{*}\left(\bar{\xi}^{h}\right), f_{*}\left(\bar{J} \bar{\xi}^{h}\right)\right) \\
& =g\left(\xi, f_{*}\left(\bar{J} \bar{\xi}^{h}\right)\right) .
\end{aligned}
$$

Now,

$$
f_{*}\left(\bar{J} \bar{\xi}^{h}\right)=\phi \circ f_{*} \bar{\xi}^{h}+\bar{\eta}\left(\bar{\xi}^{h}\right) \xi=\bar{\eta}\left(\bar{\xi}^{h}\right) \xi,
$$

so we have

$$
0=g\left(\xi, \bar{\eta}\left(\bar{\xi}^{h}\right) \xi\right)=\bar{\eta}\left(\bar{\xi}^{h}\right) g(\xi, \xi)=\bar{\eta}\left(\bar{\xi}^{h}\right) .
$$

Thus, $f_{*}\left(\bar{J} \bar{\xi}^{h}\right)=0$.

Hence, $\bar{J} \bar{\xi}^{h}$ is a vertical vector field.

(ii) Since $f: \bar{M}^{2 m} \rightarrow M^{2 n+1}$ is smooth submersion, $\bar{\eta}^{h}=\bar{\eta}_{\left.\right|_{\mathscr{H}}}$ is restriction of $\bar{\eta}$ on the horizontal distribution
$\mathscr{H}$, and $f_{*_{p}}: \mathscr{H}_{p} \rightarrow T_{f(p)} M$ is a linear isometry, for any $\bar{X}_{p} \in \mathscr{H}_{p}$, we get

$$
\begin{aligned}
\bar{\eta}_{p}^{h}\left(\bar{X}_{p}\right) & =\varepsilon \bar{g}_{p}\left(\bar{\xi}_{p}^{h}, \bar{X}_{p}\right)=g_{f(p)}\left(f_{*_{p}} \bar{\xi}_{p}^{h}, f_{*_{p}} \bar{X}_{p}\right) \\
& =g_{f(p)}\left(\xi_{f(p)}, X_{f(p)}\right)=\eta_{f(p)}\left(X_{f(p)}\right)=f_{*}^{*} \eta_{p}\left(\bar{X}_{p}\right) .
\end{aligned}
$$

Hence, pullback $f_{*}^{*} \eta=\bar{\eta}^{h}$.

Results (iii) and (iv) immediately follow from the previous results.

Example 8. Let $\left(\mathbb{R}_{2}^{4}, \bar{J}, \bar{g}\right)$ be a paracomplex pseudometric manifold and let $\left(\mathbb{R}_{1}^{3}, \phi, \xi, \eta, g\right)$ be an almost paracontact pseudometric manifold.

Define a submersion $f:\left\{\mathbb{R}_{2}^{4} ;\left(x_{1}, x_{2}, y_{1}, y_{2}\right)^{t}\right\} \rightarrow$ $\left\{\mathbb{R}_{1}^{3} ;(u, v, w)^{t}\right\}$ by

$$
\begin{array}{r}
f\left(\left(x_{1}, x_{2}, y_{1}, y_{2}\right)^{t}\right) \longmapsto\left(x_{1}+x_{2}+3 y_{1}+2 y_{2},\right. \\
3 x_{1}+2 x_{2}+y_{1}+y_{2}, \\
\left.5 x_{1}+3 x_{2}+5 y_{1}+3 y_{2}\right)^{t} .
\end{array}
$$

Then, the kernel of $f_{*}$ is

$$
\mathscr{V}=\operatorname{ker} f_{*}=\operatorname{Span}\left\{V_{1}=\frac{\partial}{\partial x_{1}}-2 \frac{\partial}{\partial x_{2}}-\frac{\partial}{\partial y_{1}}+2 \frac{\partial}{\partial y_{2}}\right\},
$$

which is the vertical distribution admitting one lightlike vector field; that is, fibre is degenerate submanifold of $\mathbb{R}_{2}^{4}$.

The horizontal distribution is

$$
\begin{gathered}
\mathscr{H}=\left(\operatorname{ker} f_{*}\right)^{\perp} \\
=\operatorname{Span}\left\{\bar{X}_{1}=\frac{\partial}{\partial x_{1}}-\frac{\partial}{\partial y_{1}}, \bar{X}_{2}=\frac{\partial}{\partial x_{2}}+2 \frac{\partial}{\partial y_{1}},\right. \\
\left.\bar{X}_{3}=2 \frac{\partial}{\partial y_{1}}+\frac{\partial}{\partial y_{2}}\right\} .
\end{gathered}
$$

For any real $k$, the horizontal characteristic vector field $\bar{\xi}^{h}$ is given by

$$
\bar{\xi}^{h}=k \frac{\partial}{\partial x_{1}}-\left(2 k-\frac{1}{3}\right) \frac{\partial}{\partial x_{2}}-(k-1) \frac{\partial}{\partial y_{1}}+\left(2 k-\frac{5}{3}\right) \frac{\partial}{\partial y_{2}},
$$

which is $f$-related to the characteristic vector field $\xi=\partial / \partial w$.

Moreover, there exists one form $\bar{\eta}=5 d x_{1}+3 d x_{2}+5 d y_{1}+$ $3 d y_{2}$ on $\left(\mathbb{R}_{2}^{4}, \bar{J}, \bar{g}\right)$ such that the submersion satisfies (39).

Example 9. Let $\left(\mathbb{R}_{3}^{6}, \bar{J}, \bar{g}\right)$ be an almost paracomplex pseudoRiemannian manifold and let $\left(\mathbb{R}_{1}^{3}, \phi, \xi, \eta, g\right)$ be an almost 
paracontact pseudo-Riemannian manifold. Consider a submersion $f:\left\{\mathbb{R}_{3}^{6} ;\left(x_{1}, x_{2}, x_{3}, y_{1}, y_{2}, y_{3}\right)^{t}\right\} \rightarrow\left\{\mathbb{R}_{1}^{3} ;(u, v, w)^{t}\right\}$, defined by

$$
\begin{aligned}
& f\left(\left(x_{1}, x_{2}, x_{3}, y_{1}, y_{2}, y_{3}\right)^{t}\right) \\
& \quad \longmapsto\left(\frac{x_{1}+x_{2}}{\sqrt{2}}, \frac{y_{1}+y_{2}}{\sqrt{2}}, \frac{y_{2}+y_{3}}{\sqrt{2}}\right)^{t} .
\end{aligned}
$$

Then, there exists one form $\bar{\eta}=\left(d x_{2}+d x_{3}\right) / \sqrt{2}$ on $\left(\mathbb{R}_{3}^{6}, \bar{J}, \bar{g}\right)$ such that (39) is satisfied. The kernel of $f_{*}$ is

$$
\begin{gathered}
\mathscr{V}=\operatorname{ker} f_{*} \\
=\operatorname{Span}\left\{V_{1}=\frac{\partial}{\partial x_{1}}-\frac{\partial}{\partial x_{2}}, V_{2}=\frac{\partial}{\partial y_{1}}-\frac{\partial}{\partial y_{2}}+\frac{\partial}{\partial y_{3}},\right. \\
\left.V_{3}=\frac{\partial}{\partial x_{3}}\right\},
\end{gathered}
$$

which is vertical distribution admitting non-lightlike vector fields; that is, the fibre is nondegenerate submanifold of $\left(\mathbb{R}_{3}^{6}, \bar{J}, \bar{g}\right)$.

The horizontal distribution is

$$
\begin{gathered}
\mathscr{H}=\operatorname{Span}\left\{\bar{X}_{1}=\frac{\partial}{\partial x_{1}}+\frac{\partial}{\partial x_{2}}, \bar{X}_{2}=-\frac{\partial}{\partial y_{1}}+\frac{\partial}{\partial y_{3}},\right. \\
\left.\bar{X}_{3}=\frac{\partial}{\partial y_{1}}+\frac{\partial}{\partial y_{2}}\right\} .
\end{gathered}
$$

Example 10. Let $\left(\mathbb{R}_{2}^{4}, \bar{J}, \bar{g}\right)$ be a paracomplex pseudometric manifold and let $\left(\mathbb{R}_{1}^{3}, \phi, \xi, \eta, g\right)$ be an almost paracontact pseudometric manifold.

Consider a submersion $f:\left\{\mathbb{R}_{2}^{4} ;\left(x_{1}, x_{2}, y_{1}, y_{2}\right)^{t}\right\} \rightarrow\left\{\mathbb{R}_{1}^{3}\right.$; $\left.(u, v, w)^{t}\right\}$, defined by

$$
f\left(\left(x_{1}, x_{2}, y_{1}, y_{2}\right)^{t}\right) \longmapsto\left(x_{1}, y_{1}, y_{2}\right)^{t} .
$$

Then, the kernel of $f_{*}$ is

$$
\mathscr{V}=\operatorname{ker} f_{*}=\operatorname{Span}\left\{V_{1}=\frac{\partial}{\partial x_{2}}\right\},
$$

which is the vertical distribution and the restriction of $\bar{g}$ to the fibres of $f$ is nondegenerate.

The horizontal distribution is

$$
\mathscr{H}=\left(\operatorname{ker} f_{*}\right)^{\perp}=\operatorname{Span}\left\{\bar{X}=\frac{\partial}{\partial x_{1}}, \bar{Y}=\frac{\partial}{\partial y_{1}}, \bar{\xi}^{h}=\frac{\partial}{\partial y_{2}}\right\} .
$$

The characteristic vector field $\xi=\partial / \partial w$ on $\mathbb{R}_{1}^{3}$ has unique horizontal lift $\bar{\xi}^{h}$, which is the characteristic vector field on horizontal distribution $\mathscr{H}$ of $\mathbb{R}_{2}^{4}$.

We also have

$$
\begin{gathered}
\bar{g}(\bar{X}, \bar{X})=g\left(f_{*} \bar{X}, f_{*} \bar{X}\right)=-1, \\
\bar{g}(\bar{Y}, \bar{Y})=g\left(f_{*} \bar{Y}, f_{*} \bar{Y}\right)=1, \\
\bar{g}\left(\bar{\xi}^{h}, \bar{\xi}^{h}\right)=g\left(f_{*} \bar{\xi}^{h}, f_{*} \bar{\xi}^{h}\right)=g(\xi, \xi)=1 .
\end{gathered}
$$

Thus, the smooth map $f$ is a pseudo-Riemannian submersion.

Moreover, we obtain that there exists a 1-form $\bar{\eta}=d x_{2}$ on $\mathbb{R}_{2}^{4}$ such that $\bar{\eta}\left(\bar{J} \bar{\xi}^{h}\right)=1, \bar{\eta}\left(\bar{\xi}^{h}\right)=0$ and the map $f$ satisfies

$$
\begin{gathered}
f_{*} \overline{J X}=\phi f_{*} \bar{X}+\bar{\eta}(\bar{X}) \xi \\
f_{*} \overline{J Y}=\phi f_{*} \bar{Y}+\bar{\eta}(\bar{Y}) \xi \\
f_{*} \bar{J}^{h}=\phi f_{*} \bar{\xi}^{h}+\bar{\eta}\left(\bar{\xi}^{h}\right) \xi .
\end{gathered}
$$

Hence, the map $f$ is a paracomplex paracontact pseudo-Riemannian submersion from $\mathbb{R}_{2}^{4}$ on to $\mathbb{R}_{1}^{3}$.

Proposition 11. Let $f: \bar{M} \rightarrow M$ be a paracomplex paracontact pseudo-Riemannian submersion and let the fibres of $f$ be pseudo-Riemannian submanifolds of $\bar{M}$. Let $\bar{X}, \bar{Y}$ be basic vector fields $f$-related to $X, Y$, respectively. Then, $\bar{J}(\bar{X})-$ $\varepsilon \bar{g}\left(\bar{X}, \bar{\xi}^{h}\right) \bar{\xi}^{h}$ is $f$-related to $\phi X$.

Proof. Since $\bar{X}$ is $f$-related to vector field $X$ on $M$, we have

$$
\begin{aligned}
\bar{\eta}(\bar{X}) & =\left\{\bar{\eta}^{v}+\bar{\eta}^{h}\right\}(\bar{X})=0+\bar{\eta}^{h}(\bar{X})=\varepsilon \bar{g}\left(\bar{X}, \bar{\xi}^{h}\right), \\
& \Longrightarrow f_{*}(\bar{J} \bar{X})=\phi X+\bar{\eta}^{h}(\bar{X}) \xi, \\
& \Longrightarrow f_{*}\left\{\bar{J} \bar{X}-\varepsilon \bar{g}\left(\bar{X}, \bar{\xi}^{h}\right) \bar{\xi}^{h}\right\}=\phi X .
\end{aligned}
$$

Hence, $\bar{J}(\bar{X})-\varepsilon \bar{g}\left(\bar{X}, \bar{\xi}^{h}\right) \bar{\xi}^{h}$ is $f$-related to $\phi X$.

Proposition 12. Let $f: \bar{M} \rightarrow M$ be a paracomplex paracontact pseudo-Riemannian submersion and let the fibres of $f$ be pseudo-Riemannian submanifolds of $\bar{M}$. Let $\mathscr{V}$ and $\mathscr{H}$ be the vertical and horizontal distributions, respectively. If $\bar{\xi}^{h}$ is the basic characteristic vector field of horizontal distribution $f$-related to the characteristic vector field $\xi$ of base manifold, then

(i) $\bar{J} \mathscr{V} \subset \overline{\mathscr{D}}^{v} \oplus\left\{\bar{J} \bar{\xi}^{h}\right\} \oplus\left\{\bar{\xi}^{h}\right\}$

(ii) $\bar{J} \mathscr{H} \subset \overline{\mathscr{D}}^{h} \oplus\left\{\bar{\xi}^{h}\right\} \oplus\left\{\bar{J} \bar{\xi}^{h}\right\}$.

Proof. (i) Let $\bar{U} \in \mathscr{V}$. Then, $\bar{U}=a \bar{U}_{\bar{I}_{\overline{\mathscr{D}}^{v}}}+b \bar{J} \bar{\xi}^{h}$, for $a, b \in$ $C^{\infty}(\bar{M})$, as $\bar{J} \bar{\xi}^{h}=\bar{\xi}^{v}$ is characteristic vector field on odd 
dimensional fibre submanifold $f^{-1}(q)$ of $\bar{M}, q \in M$. We get

$$
\begin{aligned}
\overline{J U} & =a \bar{J} \bar{U}_{\bar{I}_{\Phi^{\nu}}}+b \bar{J}^{2} \bar{\xi}^{h} \\
& =a \bar{J} \bar{U}_{\left.\right|_{\bar{\Phi}^{\nu}}}+b \bar{\xi}^{h} \in \mathscr{V} \oplus\left\{\bar{\xi}^{h}\right\}, \\
& \Longrightarrow \bar{J} \mathscr{V} \subset \mathscr{V} \oplus\left\{\bar{\xi}^{h}\right\} .
\end{aligned}
$$

Again, let $\bar{V} \in \mathscr{V} \oplus\left\{\bar{\xi}^{h}\right\}$. Then $\bar{V}=a \bar{V}_{\left.\right|_{\bar{\Phi}^{v}}}+b \bar{J} \bar{\xi}^{h}+c \bar{\xi}^{h}$, where $\bar{\eta}^{v}\left(\bar{V}_{\overline{\mid}_{\Phi^{\nu}}}\right)=0, \overline{\mathscr{D}}^{v}=\operatorname{ker} \eta^{v}, a \bar{V}_{\overline{\mid}_{\bar{D}^{v}}}+b \bar{J} \bar{\xi}^{h} \in \mathscr{V}$, and $a, b, c \in$ $C^{\infty}(\bar{M})$. We have

$$
\begin{aligned}
& \bar{J} \bar{V}=a \bar{J} \bar{V}_{\left.\right|_{\bar{\Phi}^{v}}}+b \bar{\xi}^{h}+c \bar{J} \bar{\xi}^{h} \\
& =\underbrace{\left(a \bar{J} \bar{V}_{\overline{\mid}^{v}}+c \bar{J} \bar{\xi} \bar{\xi}^{h}\right)}_{\in \mathscr{V}}+\underbrace{b \bar{\xi}^{h}}_{\in\left\{\bar{\xi}^{h}\right\}} \in \mathscr{V} \oplus\left\{\bar{\xi}^{h}\right\} .
\end{aligned}
$$

Now, by (39), we get

$$
\begin{aligned}
f_{*} \bar{J} \bar{V} & =\phi\left(f_{*} \bar{V}\right)+\bar{\eta}\left(f_{*}(\bar{V})\right) \xi \\
& =c\left\{\phi\left(f_{*} \bar{\xi}^{h}\right)+\eta(\xi) \xi\right\} \\
& =c \xi \in\{\xi\} \nsubseteq \bar{J} \mathscr{V} .
\end{aligned}
$$

We get $\bar{J} V \notin \mathscr{V}$.

Hence, $\bar{J} \mathscr{V} \subset \mathscr{V} \oplus\left\{\bar{\xi}^{h}\right\}$; that is, $\bar{J} \mathscr{V} \subset \overline{\mathscr{D}}^{v} \oplus\left\{\bar{J} \bar{\xi}^{h}\right\} \oplus\left\{\bar{\xi}^{h}\right\}$.

(ii) Let $\bar{X}=a \bar{X}_{\left.\right|_{\overline{\mathscr{D}}^{h}}}+b \bar{\xi}^{h} \in \mathscr{H}$, where $\mathscr{H}=\overline{\mathscr{D}}^{h} \oplus$ $\left\{\bar{\xi}^{h}\right\}, \operatorname{ker} \bar{\eta}^{h}=\overline{\mathscr{D}}^{h}$, and $a, b \in C^{\infty}(\bar{M})$. Then

$$
\bar{J} \bar{X}=a \bar{J} \bar{X}_{\left.\right|_{\bar{\Phi}^{h}}}+b \bar{J} \bar{\xi}^{h} \in \mathscr{H} \oplus\left\{\bar{J} \bar{\xi}^{h}\right\},
$$

which implies that $\bar{J} \mathscr{H} \subset \mathscr{H} \oplus\left\{\bar{J} \bar{\xi}^{h}\right\}$.

Again, let $\bar{Y} \in \mathscr{H} \oplus\left\{\bar{J} \bar{\xi}^{h}\right\}$. Then, $\bar{Y}=a \bar{Y}_{\left.\right|_{\bar{g}^{h}}}+b \bar{\xi}^{h}+c \bar{J} \bar{\xi}^{h} \notin \mathscr{H}$, for $a, b, c \in C^{\infty}(\bar{M})$. We have

$$
\begin{aligned}
\bar{J} \bar{Y} & =a \bar{J} \bar{Y}_{\left.\right|_{\overline{\mathscr{D}}^{h}}}+b \bar{J} \bar{\xi}^{h}+c \bar{J}^{2} \bar{\xi}^{h} \\
& =a \bar{J} \bar{Y}_{\left.\right|_{\overline{\mathscr{S}}^{h}}}+b \bar{J} \bar{\xi}^{h}+c \bar{\xi}^{h} \\
& =\bar{Z}+b \bar{J} \bar{\xi}^{h} \in \mathscr{H} \oplus\left\{\bar{J} \bar{\xi}^{h}\right\},
\end{aligned}
$$

for some $\bar{Z}=a \bar{J} \bar{Y}_{\bar{D}_{\bar{D}^{h}}}+c \bar{\xi}^{h} \in \mathscr{H}$.

We obtain $\bar{J} \bar{Y} \notin \mathscr{H}$.

Hence, $\bar{J} \mathscr{H} \subset \mathscr{H} \oplus\left\{\bar{J}\left(\bar{\xi}^{h}\right)\right\}$; that is, $\bar{J} \mathscr{H} \subset \overline{\mathscr{D}}^{h} \oplus\left\{\bar{\xi}^{h}\right\} \oplus$ $\left\{\bar{J} \bar{\xi}^{h}\right\}$.

Example 13. Let $\left(\mathbb{R}_{3}^{6}, \bar{J}, \bar{g}\right)$ be an almost paracomplex pseudoRiemannian manifold and let $\left(\mathbb{R}_{1}^{3}, \phi, \xi, \eta, g\right)$ be an almost paracontact pseudo-Riemannian manifold. Consider a submersion $f:\left\{\mathbb{R}_{3}^{6} ;\left(x_{1}, x_{2}, x_{3}, y_{1}, y_{2}, y_{3}\right)^{t}\right\} \rightarrow\left\{\mathbb{R}_{1}^{3} ;(u, v, w)^{t}\right\}$, defined by

$$
f\left(\left(x_{1}, x_{2}, x_{3}, y_{1}, y_{2}, y_{3}\right)^{t}\right) \longmapsto\left(\frac{x_{1}+x_{2}}{\sqrt{2}}, \frac{y_{1}+y_{2}}{\sqrt{2}}, y_{3}\right)^{t} .
$$

Then, the kernel of $f_{*}$ is

$$
\begin{gathered}
\mathscr{V}=\operatorname{ker} f_{*} \\
=\operatorname{Span}\left\{V_{1}=\frac{\partial}{\partial x_{1}}-\frac{\partial}{\partial x_{2}}, V_{2}=\frac{\partial}{\partial y_{1}}-\frac{\partial}{\partial y_{2}},\right. \\
\left.\bar{\xi}^{v}=\frac{\partial}{\partial x_{3}}\right\}
\end{gathered}
$$

which is the vertical distribution and the restriction of $\bar{g}$ to the fibres of $f$ is nondegenerate.

The horizontal distribution is

$$
\begin{gathered}
\mathscr{H}=\left(\operatorname{ker} f_{*}\right)^{\perp} \\
=\operatorname{Span}\left\{\bar{X}_{1}=\frac{\partial}{\partial x_{1}}+\frac{\partial}{\partial x_{2}}, \bar{X}_{2}=\frac{\partial}{\partial y_{1}}+\frac{\partial}{\partial y_{2}},\right. \\
\left.\bar{\xi}^{h}=\frac{\partial}{\partial y_{3}}\right\} .
\end{gathered}
$$

The characteristic vector field $\xi=\partial / \partial w$ on $\mathbb{R}_{1}^{3}$ has unique horizontal lift $\bar{\xi}^{h}$, which is the characteristic vector field on the horizontal distribution $\mathscr{H}$ of $\mathbb{R}_{3}^{6}$.

We also have

$$
\begin{gathered}
\bar{g}\left(\bar{X}_{1}, \bar{X}_{1}\right)=g\left(f_{*} \bar{X}_{1}, f_{*} \bar{X}_{1}\right)=-2, \\
\bar{g}\left(\bar{X}_{2}, \bar{X}_{2}\right)=g\left(f_{*} \bar{X}_{2}, f_{*} \bar{X}_{2}\right)=2, \\
\bar{g}\left(\bar{\xi}^{h}, \bar{\xi}^{h}\right)=g\left(f_{*} \bar{\xi}^{h}, f_{*} \bar{\xi}^{h}\right)=g(\xi, \xi)=1 .
\end{gathered}
$$

Thus, the smooth map $f$ is a pseudo-Riemannian submersion.

Also, we obtain that there exists a 1-form $\bar{\eta}=d x_{3}$ on $\mathbb{R}_{3}^{6}$ such that $\bar{\eta}\left(\bar{J} \bar{\xi}^{h}\right)=1, \bar{\eta}\left(\bar{\xi}^{h}\right)=0$ and the map $f$ satisfies

$$
\begin{gathered}
f_{*} \overline{J X}_{1}=\phi f_{*} \bar{X}_{1}+\bar{\eta}\left(\bar{X}_{1}\right) \xi, \\
f_{*} \bar{J} \bar{X}_{2}=\phi f_{*} \bar{X}_{2}+\bar{\eta}\left(\bar{X}_{2}\right) \xi, \\
f_{*} \bar{J} \bar{\xi}^{h}=\phi f_{*} \bar{\xi}^{h}+\bar{\eta}\left(\bar{\xi}^{h}\right) \xi .
\end{gathered}
$$

Hence, the map $f$ is a paracomplex paracontact pseudoRiemannian submersion from $\mathbb{R}_{3}^{6}$ onto $\mathbb{R}_{1}^{3}$.

Moreover, we observe that, for this submersion $f$, we have

$$
\bar{J} \mathscr{V} \subset \mathscr{V} \oplus\left\{\bar{\xi}^{h}\right\}, \quad \bar{J} \mathscr{H} \subset \mathscr{H} \oplus\left\{\bar{J} \bar{\xi}^{h}\right\},
$$

which verifies Proposition 12. 
Proposition 14. Let $f: \bar{M} \rightarrow M$ be a paracomplex paracontact pseudo-Riemannian submersion and let the fibres of $f$ be pseudo-Riemannian submanifolds of $\bar{M}$. Let $\bar{X}, \bar{Y}$ be basic vector fields $f$-related to $X, Y$, respectively. Let $F$ and $\Phi$ be the second fundamental forms and let $\bar{\nabla}$ and $\nabla$ be the LeviCivita connection on the total manifold $\bar{M}$ and base manifold $M$, respectively. Then, one has

(i) $f_{*}\left(\left(\bar{\nabla}_{\bar{X}} \bar{J}\right) \bar{Y}\right)=\left(\nabla_{X} \phi\right) Y+\varepsilon g\left(Y, \nabla_{X} \xi\right) \xi+\eta(Y) \nabla_{X} \xi$,

(ii) $F=f_{*}^{*} \Phi+\varepsilon \bar{\eta} \otimes \bar{\eta}$,

(iii) $f_{*}\left(\left(\bar{\nabla}_{\bar{X}} F\right)(\bar{Y}, \bar{Z})\right)=\left(\nabla_{X} \Phi\right)(Y, Z)+\eta(Y) g\left(Z, \nabla_{X} \xi\right)+$ $\eta(Z) g\left(Y, \nabla_{X} \xi\right)$.

Proof. (i) In view of Definition 2 and Proposition 11, we have

$$
\begin{aligned}
f_{*}\left(\left(\bar{\nabla}_{\bar{X}} \bar{J}\right) \bar{Y}\right)= & f_{*}\left(\bar{\nabla}_{\bar{X}}(\bar{J} \bar{Y})-\bar{J}\left(\bar{\nabla}_{\bar{X}} \bar{Y}\right)\right) \\
= & \nabla_{X}\left(f_{*}(\bar{J} \bar{Y})\right)-f_{*}(\bar{J}(\bar{\nabla} \bar{X} \bar{Y})) \\
= & \nabla_{X}(\phi Y)+\nabla_{X}(\eta(Y) \xi)-\phi\left(\nabla_{X} Y\right) \\
& -\eta\left(\nabla_{X} Y\right) \xi \\
= & \left(\nabla_{X} \phi\right) Y+\nabla_{X}(\varepsilon g(Y, \xi) \xi)-\eta\left(\nabla_{X} Y\right) \xi \\
= & \left(\nabla_{X} \phi\right) Y+\varepsilon g\left(\nabla_{X} Y, \xi\right) \xi+\varepsilon g\left(Y, \nabla_{X} \xi\right) \xi \\
& +\varepsilon g(Y, \xi) \nabla_{X} \xi-\varepsilon g\left(\nabla_{X} Y, \xi\right) \xi \\
= & \left(\nabla_{X} \phi\right) Y+\varepsilon g\left(Y, \nabla_{X} \xi\right) \xi+\eta(Y) \nabla_{X} \xi .
\end{aligned}
$$

(ii) Since $f_{*}^{*} \Phi$ is pullback of $\Phi$ through the linear map $f_{*}$, we get

$$
\begin{aligned}
f_{*}^{*} \Phi(\bar{X}, \bar{Y}) & =\Phi(X, Y) \circ f=g(X, \phi Y) \circ f \\
& =\bar{g}(\bar{X}, \bar{J} \bar{Y})-\varepsilon \bar{\eta}(\bar{Y}) \bar{\eta}(\bar{X}) \\
& =F(\bar{X}, \bar{Y})-\varepsilon \bar{\eta}(\bar{X}) \bar{\eta}(\bar{Y}),
\end{aligned}
$$

which implies $F=f_{*}^{*} \Phi+\varepsilon \bar{\eta} \otimes \bar{\eta}$.

(iii) By (23), we have

$$
f_{*}\left(\left(\bar{\nabla}_{\bar{X}} F\right)(\bar{Y}, \bar{Z})\right)=g\left(f_{*}(\bar{Y}), f_{*}\left(\left(\bar{\nabla}_{X} \bar{J}\right) \bar{Z}\right)\right) .
$$

Now, using (i) in the above equation, we get (iii).

Theorem 15. Let $f: \bar{M} \rightarrow M$ be a paracomplex paracontact pseudo-Riemannian submersion and let the fibres of $f$ be pseudo-Riemannian submanifolds of $\bar{M}$. Let $\bar{X}, \bar{Y}$ be basic vector fields $f$-related to $X, Y$, respectively. If the total space is para-Hermitian manifold, then the almost paracontact structure of base space is normal.

Moreover, if the almost paracontact structure of base space is normal, then the Nijenhuis tensor of total space is vertical.

Proof. The Nijenhuis tensors $N_{\bar{J}}$ and $N_{\phi}$ of almost paracomplex structure $\bar{J}$ and almost paracontact structure $\phi$ are, respectively, defined by (8) and (11).
Using Definition 2 and properties of Sections 2.1 and 2.2, we get the following identity:

$$
\begin{aligned}
f_{*}\left(N_{\bar{J}}(\bar{X}, \bar{Y})\right)= & N^{(1)}(X, Y)+2 d \eta(\phi X, Y) \xi \\
& -2 d \eta(\phi Y, X) \xi \\
& +2 \eta(X) d \eta(\xi, Y) \xi-2 \eta(Y) d \eta(\xi, X) \xi \\
& -\eta(Y) N^{(3)}(X)+\eta(X) N^{(3)}(Y) .
\end{aligned}
$$

Using (12), (13), (14), and (15), (80) reduces to

$$
\begin{aligned}
f_{*}\left(N_{\bar{J}}(\bar{X}, \bar{Y})\right)= & N^{(1)}(X, Y)+N^{(2)}(X, Y) \xi \\
& +\eta(X) N^{(4)}(Y) \xi \\
& -\eta(Y) N^{(4)}(X) \xi-\eta(Y) N^{(3)}(X) \\
& +\eta(X) N^{(3)}(Y) .
\end{aligned}
$$

Since $N_{\bar{J}}(\bar{X}, \bar{Y})=0$, it follows from (81) that tensors $N^{(1)}, N^{(2)}, N^{(3)}$, and $N^{(4)}$ vanish together.

Hence, the almost paracontact structure of base space is normal.

Conversely, let the almost paracontact structure of the base space be normal.

Then, (81) implies that $f_{*}\left(N_{\bar{J}}(\bar{X}, \bar{Y})\right)=0$.

Hence, $N_{\bar{J}}(\bar{X}, \bar{Y})$ is vertical.

Corollary 16. Let $f: \bar{M} \rightarrow M$ be a paracomplexparacontact pseudo-Riemannian submersion and let the fibres of $f$ be pseudo-Riemannian submanifolds of $\bar{M}$. Let $\bar{X}, \bar{Y}$ be basic vector fields $f$-related to $X, Y$, respectively. Let the total space be para-Hermitian manifold and $N^{(1)}$ vanishes. Then, the base space is paracontact pseudometric manifold if and only if $\xi$ is killing.

Proof. Let the total space be para-Hermitian and $N^{(1)}$ vanishes. Then, from (80), we have

$$
\begin{aligned}
0= & 2 d \eta(\phi X, Y) \xi-2 d \eta(\phi Y, X) \xi+2 \eta(X) d \eta(\xi, Y) \xi \\
& -2 \eta(Y) d \eta(\xi, X) \xi-\eta(Y)\left(\mathscr{L}_{\xi} \phi\right) X+\eta(X)\left(\mathscr{L}_{\xi} \phi\right) Y .
\end{aligned}
$$

If $\xi$ is killing, then we have $\mathscr{L}_{\xi} \phi=0$. It immediately follows from (82) that

$$
\begin{gathered}
d \eta(\phi X, Y)-\eta(\phi Y, X)+\eta(X) d \eta(\xi, Y) \\
-\eta(Y) d \eta(\xi, X)=0 .
\end{gathered}
$$

In view of (6) and (7), the above equation gives $d \eta=\Phi$.

Conversely, let the base space be paracontact. Then, $d \eta=$ $\Phi$.

Using (6), (7), and (82), we get $\mathscr{L}_{\xi} \phi=0$.

Hence, the characteristic vector field $\xi$ is killing. 
Theorem 17. Let $f: \bar{M} \rightarrow M$ be a paracomplex paracontact pseudo-Riemannian submersion and let the fibres of $f$ be pseudo-Riemannian submanifolds of $\bar{M}$. Let $\bar{X}, \bar{Y}$ be basic vector fields $f$-related to $X, Y$, respectively. If the total space is para-Kähler, then the base space is paracosymplectic. The converse is true if $\bar{\nabla} \bar{X} \bar{J}$ is vertical.

Proof. We have, for any $X, Y \in \Gamma(T M),\left(\nabla_{X} \phi\right) Y=0$, which gives $g\left(Z,\left(\nabla_{X} \phi\right) Y\right)=0$, for any $Z \in \Gamma(T M)$.

From Proposition 14, we have

$$
\begin{aligned}
& \left\{g\left(Z,\left(\nabla_{X} \phi\right) Y\right)+\varepsilon \eta(Y)\left(\nabla_{X} \eta\right) Z+\varepsilon \eta(Z)\left(\nabla_{X} \eta\right) Y\right\} \circ f \\
& \quad=\bar{g}\left(\bar{Z},\left(\bar{\nabla}_{\bar{X}} \bar{J}\right) \bar{Y}\right) .
\end{aligned}
$$

Let $\bar{\nabla} \bar{J}=0$; that is, the total space is para-Kähler. Then, from (84), we obtain $\nabla \phi=0$ and $\nabla \eta=0$. Hence, the base space is paracosymplectic.

Again, let $\left(\nabla_{X} \phi\right) Y=0$ and $\nabla \eta=0$. Then, $\bar{g}(\bar{Z},(\bar{\nabla} \bar{X} \bar{J}) \bar{Y})=$ 0 , which implies that $(\bar{\nabla} \bar{X} \bar{J}) \bar{Y}$ is a vertical vector field.

Theorem 18. Let $f: \bar{M} \rightarrow M$ be a paracomplex paracontact pseudo-Riemannian submersion and let the fibres of $f$ be pseudo-Riemannian submanifolds of $\bar{M}$. Let $\bar{X}, \bar{Y}$, and $\bar{Z}$ be basic vector fields $f$-related to $X, Y$, and $Z$, respectively. If $\eta(X) Z(\eta(Y))+\eta(Y) X(\eta(Z))-\eta(X) Y(\eta(Z))=0$, then the total space is almost para-Kähler if and only if the base space $M$ is an almost paracosymplectic manifold.

Proof. We have the following equation:

$$
\begin{aligned}
3 d F( & \bar{X}, \bar{Y}, \bar{Z}) \\
= & 3\left(f_{*}^{*} d \Phi\right)(\bar{X}, \bar{Y}, \bar{Z})+2 \varepsilon \bar{\eta}(\bar{Z}) d \bar{\eta}(\bar{X}, \bar{Y}) \\
& -2 \varepsilon \bar{\eta}(\bar{Y}) d \bar{\eta}(\bar{X}, \bar{Z}) \\
& +2 \varepsilon \bar{\eta}(\bar{X}) d \bar{\eta}(\bar{Y}, \bar{Z})+2 \varepsilon \bar{\eta}(\bar{X}) \bar{Z}(\bar{\eta}(\bar{Y})) \\
& +2 \varepsilon \bar{\eta}(\bar{Y}) \bar{X}(\bar{\eta}(\bar{Z})) \\
& -2 \varepsilon \bar{\eta}(\bar{X}) \bar{Y}(\bar{\eta}(\bar{Z})) .
\end{aligned}
$$

If $d \eta=0, d \Phi=0$, and $\eta(X) Z(\eta(Y))+\eta(Y) X(\eta(Z))-$ $\eta(X) Y(\eta(Z))=0$, then, from (85), we have $d F=0$. Hence, the total space is almost para-Kähler.

Conversely, let $d F=0$ and $\eta(X) Z(\eta(Y))+\eta(Y) X(\eta(Z))-$ $\eta(X) Y(\eta(Z))=0$.

By using the above equation in (85), we have $d \eta=0$ and $d \Phi=0$.

Hence, the base space is almost paracosymplectic.

Now, we investigate the properties of fundamental tensors $\mathscr{T}$ and $\mathscr{A}$ of a pseudo-Riemannian submersion.

Lemma 19. Let $f: \bar{M} \rightarrow M$ be a paracomplex paracontact pseudo-Riemannian submersion from a para-Kähler manifold $\bar{M}$ onto an almost paracontact pseudometric manifold $M$ and let the fibres of $f$ be pseudo-Riemannian submanifolds of $\bar{M}$. Then, for any horizontal vector fields $\bar{X}, \bar{Y}$ and for any vertical vector fields $U, V$ on $\bar{M}$, one has
(i) $\mathscr{A}_{\bar{X}}(\bar{J} \bar{Y})=\bar{J}\left(\mathscr{A}_{\bar{X}} \bar{Y}\right)$,
(ii) $\mathscr{A}_{\bar{J} \bar{X}}(\bar{Y})=\bar{J}\left(\mathscr{A}_{\bar{X}} \bar{Y}\right)$,
(iii) $\mathscr{T}_{U}(\bar{J} V)=\bar{J}\left(\mathscr{T}_{U} V\right)$,
(iv) $\mathscr{T}_{\bar{J} U} V=\bar{J}\left(\mathscr{T}_{U} V\right)$.

Proof. The proof follows using similar steps as in Lemmas 3 and 4 of [13], so we omit it.

Lemma 20. Let $f: \bar{M} \rightarrow M$ be a paracomplex paracontact pseudo-Riemannian submersion from a para-Kähler manifold $\bar{M}$ onto an almost paracontact pseudometric manifold $M$ and let the fibres of $f$ be pseudo-Riemannian submanifolds of $\bar{M}$. Then, for any vector fields $E, F$ on $\bar{M}$, one has
(i) $\mathscr{A}_{E}(\bar{J} F)=\bar{J}\left(\mathscr{A}_{E} F\right)$,
(ii) $\mathscr{T}_{E}(\bar{J} F)=\bar{J}\left(\mathscr{T}_{E} F\right)$.

Proof. The proof follows from (37) and (38).

Theorem 21. Let $f: \bar{M} \rightarrow M$ be a paracomplex paracontact pseudo-Riemannian submersion from a para-Kähler manifold $\bar{M}$ onto an almost paracontact pseudometric manifold $M$ and let the fibres of $f$ be pseudo-Riemannian submanifolds of $\bar{M}$. Then, the horizontal distribution is integrable.

Proof. For any vertical vector field $U$, we have

$$
\begin{aligned}
& \bar{g}\left(\bar{J}\left(\mathscr{A}_{\bar{X}} \bar{Y}\right), U\right)=\bar{g}\left(\mathscr{A}_{\bar{X}} \bar{J} \bar{Y}, U\right) \\
& =-\bar{g}\left(\bar{J} \bar{Y}, \mathscr{A}_{\bar{X}} U\right) \\
& =-\bar{g}\left(\bar{J} \bar{Y}, h\left(\bar{\nabla}_{U} \bar{X}\right)\right) \\
& =\bar{g}\left(\bar{Y}, h\left(\bar{J}\left(\bar{\nabla}_{U} \bar{X}\right)\right)\right) \\
& =\bar{g}\left(\bar{Y}, h\left\{\left(-\bar{\nabla}_{U} \bar{J}\right) \bar{X}\right.\right. \\
& \left.\left.+\bar{\nabla}_{U}(\bar{J} \bar{X})\right\}\right) \\
& =\bar{g}\left(\bar{Y}, h\left\{\bar{\nabla}_{U}(\overline{J X})\right\}\right)=\bar{g}\left(\bar{Y}, \mathscr{A}_{\bar{J} \bar{X}} U\right) \\
& =-\bar{g}\left(\mathscr{A}_{\bar{J} \bar{X}} \bar{Y}, U\right)=-\bar{g}\left(\bar{J}\left(\mathscr{A}_{\bar{X}} \bar{Y}\right), U\right) \text {. }
\end{aligned}
$$

Thus $\bar{g}\left(\bar{J}\left(\mathscr{A}_{\bar{X}} \bar{Y}\right), U\right)=0$, which is true for all $\bar{X}$ and $\bar{Y}$.

So, $\mathscr{A}_{\bar{X}} \bar{Y}=0$.

Hence, the horizontal distribution is integrable.

Theorem 22. Let $f: \bar{M} \rightarrow M$ be a paracomplex paracontact pseudo-Riemannian submersion from a para-Kähler manifold $\bar{M}$ onto an almost paracontact pseudometric manifold $M$ and let the fibres of $f$ be pseudo-Riemannian submanifolds of $\bar{M}$. Then, the submersion is an affine map on $\mathscr{H}$. 
Proof. The second fundamental form of $f$ is defined by

$$
\left(\bar{\nabla} f_{*}\right)(E, F)=\left(\nabla_{E}^{f} f_{*}(F)\right) \circ f-f_{*}\left(\bar{\nabla}_{E} F\right),
$$

where $E, F \in \Gamma(T \bar{M})$ and $\nabla^{f}$ is pullback connection of LeviCivita connection $\nabla$ on $M$ with respect to $f$.

We have, for any $\bar{X}, \bar{Y} \in \mathscr{H}$,

$$
\left(\bar{\nabla} f_{*}\right)(\bar{X}, \bar{Y})=\left(\nabla_{\bar{X}}^{f} f_{*}(\bar{Y})\right) \circ f-f_{*}(\bar{\nabla} \bar{X} \bar{Y}) .
$$

By using Lemma 1 , we have $f_{*}(h(\bar{\nabla} \bar{X} \bar{Y}))=\left(\nabla_{X} Y\right) \circ f$, which implies $\bar{\nabla} f_{*}=0$.

Hence, the submersion $f$ is an affine map on $\mathscr{H}$.

Theorem 23. Let $f: \bar{M} \rightarrow M$ be a paracomplex paracontact pseudo-Riemannian submersion from a para-Hermitian manifold $\bar{M}$ onto an almost paracontact pseudometric manifold $M$ and let the fibres of $f$ be pseudo-Riemannian submanifolds of $\bar{M}$. Then, the submersion is an affine map on $\mathscr{V}$ if and only if the fibres of $f$ are totally geodesic.

Proof. We have, for any $U, V \in \mathscr{V}$,

$$
\left(\bar{\nabla} f_{*}\right)(U, V)=-f_{*}\left(h\left(\bar{\nabla}_{U} V\right)\right),
$$

which, in view of (27), gives

$$
\left(\bar{\nabla} f_{*}\right)(U, V)=-f_{*}\left(\mathscr{T}_{U} V\right)
$$

Let the fibres of $f$ be totally geodesic. Then, $\mathscr{T}=0$. Consequently, from the above equation, we have $\bar{\nabla} f_{*}=0$.

Thus, the map $f$ is affine on $\mathscr{V}$.

Conversely, let the submersion $f$ be an affine map on $\mathscr{V}$. Then, $\bar{\nabla} f_{*}=0$, which implies $\mathscr{T}=0$.

Hence, the fibres of $f$ are totally geodesic.

Theorem 24. Let $f: \bar{M} \rightarrow M$ be a paracomplex paracontact pseudo-Riemannian submersion from a para-Hermitian manifold $\bar{M}$ onto an almost paracontact pseudometric manifold $M$ and let the fibres of $f$ be pseudo-Riemannian submanifolds of $\bar{M}$. Then, the submersion is an affine map if and only if $h\left(\bar{\nabla}_{E} h F\right)+\mathscr{A}_{h E} v F+\mathscr{T}_{v E} v F$ is $f$-related to $\nabla_{X} Y$, for any $E, F \in$ $\Gamma(T \bar{M})$.

Proof. For any $E, F \in \Gamma(T \bar{M})$ with $f_{*} h E=X \circ f$ and $f_{*} v F=$ $Y \circ f$, we have

$$
\begin{array}{r}
\left(\bar{\nabla} f_{*}\right)(E, F)=\left(\nabla_{f_{*} h E}\left(f_{*} h F\right)\right) \circ f-f_{*}\left(h\left(\bar{\nabla}_{E} F\right)\right) \\
=\left(\nabla_{X} Y\right) \circ f-f_{*}\left(h \left(\bar{\nabla}_{h E} h F+\bar{\nabla}_{h E} v F\right.\right. \\
\left.\left.+\bar{\nabla}_{v E} h F+\bar{\nabla}_{v E} h F\right)\right) .
\end{array}
$$

By using (27) and (31) in the above equation, we have

$$
\begin{gathered}
\left(\bar{\nabla} f_{*}\right)(E, F)=\left(\nabla_{X} Y\right) \circ f-f_{*}\left(h\left(\bar{\nabla}_{E} h F\right)+\mathscr{A}_{h E} v F\right. \\
\left.+\mathscr{T}_{v E} v F\right) .
\end{gathered}
$$

Let the submersion map be affine. Then, for any $E, F \in$ $\Gamma(T \bar{M}),\left(\bar{\nabla} f_{*}\right)(E, F)=0$. Equation (92) implies $\left(\nabla_{X} Y\right) \circ f=$ $f_{*}\left(h\left(\bar{\nabla}_{E} h F\right)+\mathscr{A}_{h E} v F+\mathscr{T}_{v E} h F\right)$.

Conversely, let $h\left(\bar{\nabla}_{E} F\right)+\mathscr{A}_{h E} v F+\mathscr{T}_{v E} h F$ be $f$-related to $\nabla_{X} Y$, for any $E, F \in \Gamma(T \bar{M})$. Then, from (92), we have $\left(\bar{\nabla} f_{*}\right)(E, F)=0$.

Hence, the submersion map $f$ is affine.

\section{Curvature Properties}

In this section, the paraholomorphic bisectional curvatures and paraholomorphic sectional curvatures of total manifold, base manifold, and fibres of paracomplex paracontact pseudo-Riemannian submersion and their curvature properties are studied.

Let $f: \bar{M} \rightarrow M$ be a paracomplex paracontact pseudoRiemannian submersion from an almost para-Hermitian manifold $(\bar{M}, \bar{J}, \bar{g})$ onto an almost paracontact pseudometric manifold $(M, \phi, \xi, \eta, g)$.

Suppose that the vector fields $E, F$ span the 2dimensional plane at point $p$ of $\bar{M}$ and let $\overline{\mathscr{R}}$ be the Riemannian curvature tensor of $\bar{M}$. The paraholomorphic bisectional curvature $\bar{B}(E, F)$ of $\bar{M}$ for any pair of nonzero non-lightlike vector fields $E, F$ on $\bar{M}$ is defined by the formula

$$
\bar{B}(E, F)=\frac{\overline{\mathscr{R}}(E, \bar{J} E, F, \bar{J} F)}{\bar{g}(E, E) \bar{g}(F, F)} .
$$

For a nonzero non-lightlike vector field $E$, the vector field $\bar{J} E$ is also non-lightlike and $\{E, \bar{J} E\}$ span the 2-dimensional plane. Then the paraholomorphic sectional curvature $\bar{H}(E)$ is defined as

$$
\bar{H}(E)=\bar{B}(E, E)=\frac{\overline{\mathscr{R}}(E, \bar{J} E, E, \bar{J} E)}{\bar{g}(E, E) \bar{g}(E, E)} .
$$

The curvature properties of Riemannian submersion and semi-Riemannian submersion have been extensively studied in the work of O'Neill [1] and Gray [3].

Let $\bar{B}^{h}$ and $\bar{B}^{v}$ be the paraholomorphic bisectional curvatures of horizontal and vertical spaces, respectively. Let $\bar{H}^{h}$ and $\bar{H}^{v}$ be the paraholomorphic sectional curvatures of horizontal and vertical spaces, respectively. Let $B$ and $H$ be the paraholomorphic bisectional and sectional curvatures of the base manifold, respectively.

Proposition 25. Let $f: \bar{M} \rightarrow M$ be a paracomplex paracontact pseudo-Riemannian submersion from an almost para-Hermitian manifold onto an almost paracontact pseudometric manifold and let the fibres of $f$ be pseudo-Riemannian submanifolds of $\bar{M}$. Let $U, V$ be non-lightlike unit vertical vector fields and let $\bar{X}, \bar{Y}$ be non-lightlike unit horizontal vector fields on $\bar{M}$. Then, one has

$$
\begin{aligned}
\bar{B}(U, V)= & \bar{B}^{v}(U, V)+\bar{g}\left(\mathscr{T}_{U}(\bar{J} V), \mathscr{T}_{\bar{J} U} V\right) \\
& -\bar{g}\left(\mathscr{T}_{\bar{\jmath} U}(\bar{J} V), \mathscr{T}_{U} V\right),
\end{aligned}
$$




$$
\begin{aligned}
\bar{B}(\bar{X}, U)= & \bar{g}\left(\left(\bar{\nabla}_{U} \mathscr{A}\right)_{\bar{X}} \bar{J} \bar{X}, \bar{J} U\right)-\bar{g}\left(\mathscr{A}_{\bar{X}} \bar{J} U, \mathscr{A}_{\bar{J} \bar{X}} U\right) \\
& +\bar{g}\left(\mathscr{A}_{\bar{X}} U, \mathscr{A}_{\bar{J} \bar{X}} \bar{J} U\right)-\bar{g}\left(\left(\bar{\nabla}_{\bar{J} U} \mathscr{A}\right)_{\bar{X}} \bar{J} \bar{X}, U\right) \\
& +\bar{g}\left(\mathscr{T}_{\bar{J} U} \bar{X}, \mathscr{T}_{U}(\bar{J} \bar{X})\right) \\
& -\bar{g}\left(\mathscr{T}_{U} \bar{X}, \mathscr{T}_{\bar{J} U}(\bar{J} \bar{X})\right) \\
\bar{B}(\bar{X}, \bar{Y})= & \bar{B}^{h}(\bar{X}, \bar{Y})-2 \bar{g}\left(\mathscr{A}_{\bar{X}}(\bar{J} \bar{X}), \mathscr{A}_{\bar{Y}}(\bar{J} \bar{Y})\right) \\
& +\bar{g}\left(\mathscr{A}_{\bar{J} \bar{X}} \bar{Y}, \mathscr{A}_{\bar{X}}(\bar{J} \bar{Y})\right) \\
& -\bar{g}\left(\mathscr{A}_{\bar{X}} \bar{Y}, \mathscr{A}_{\bar{J} \bar{X}}(\bar{J} \bar{Y})\right) .
\end{aligned}
$$

Proof. Using Definitions (93) and (94) of paraholomorphic sectional curvature and fundamental equations of submersion obtained by O'Neill [1], we have (95), (96), and (97).

Corollary 26. Let $f: \bar{M} \rightarrow M$ be a paracomplex paracontact pseudo-Riemannian submersion from an almost para-Hermitian manifold onto an almost paracontact pseudometric manifold. If the fibres of $f$ are totally geodesic pseudoRiemannian submanifolds of $\bar{M}$, then for any non-lightlike unit vertical vector fields $U$ and $V$, one has

$$
\bar{B}(U, V)=\bar{B}^{v}(U, V) .
$$

Corollary 27. Let $f: \bar{M} \rightarrow M$ be a paracomplex paracontact pseudo-Riemannian submersion from an almost paraHermitian manifold onto an almost paracontact pseudometric manifold and let the fibres of $f$ be totally geodesic pseudoRiemannian submanifolds of $\bar{M}$. If the horizontal distribution is integrable, then, for any non-lightlike unit horizontal vector fields $\bar{X}$ and $\bar{Y}$, one has

$$
\bar{B}(\bar{X}, \bar{Y})=\bar{B}^{h}(\bar{X}, \bar{Y}) .
$$

Proposition 28. Let $f: \bar{M} \rightarrow M$ be a paracomplex paracontact pseudo-Riemannian submersion from an almost para-Hermitian manifold onto an almost paracontact pseudometric manifold and let the fibres of $f$ be pseudo-Riemannian submanifolds of $\bar{M}$. Let $U$ and $\bar{X}$ be non-lightlike unit vertical vector field and non-lightlike unit horizontal vector field, respectively. Then, one has

$$
\begin{gathered}
\bar{H}(U)=\bar{H}^{v}(U)+\left\|\mathscr{T}_{U}(\bar{J} U)\right\|^{2}-\bar{g}\left(\mathscr{T}_{\bar{J} U}(\bar{J} U), \mathscr{T}_{U} U\right), \\
\bar{H}(\bar{X})=H(X) \circ f-3\left\|\mathscr{A}_{\bar{X}}(\bar{J} \bar{X})\right\|^{2} .
\end{gathered}
$$

Proof. The proof is straightforward. If we take $U=V$ in (95) and $\bar{X}=\bar{Y}$ in (97), we have (98) and (99).

Corollary 29. Let $f: \bar{M} \rightarrow M$ be a paracomplex paracontact pseudo-Riemannian submersion from an almost para-Hermitian manifold onto an almost paracontact pseudometric manifold. If the fibres of $f$ are totally geodesic pseudoRiemannian submanifolds of $\bar{M}$, then the total manifold and fibres of $f$ have the same paraholomorphic sectional curvatures.
Proof. Since the fibres are totally geodesic, $\mathscr{T}=0$; consequently we have

$$
\bar{H}(U)=\bar{H}^{v}(U) .
$$

Corollary 30. Let $f: \bar{M} \rightarrow M$ be a paracomplex paracontact pseudo-Riemannian submersion from an almost paraHermitian manifold onto an almost paracontact pseudometric manifold and let the fibres of $f$ be totally geodesic pseudoRiemannian submanifolds of $\bar{M}$. If the horizontal distribution is integrable, then the base manifold and horizontal distribution have the same paraholomorphic sectional curvatures.

Proof. Since the horizontal distribution is integrable, $\mathscr{A}=0$; consequently, we have

$$
\bar{H}(\bar{X})=H(X) \circ f .
$$

Theorem 31. Let $f: \bar{M}^{m} \rightarrow M^{n}$ be a paracomplex paracontact pseudo-Riemannian submersion from a paraKähler manifold $\bar{M}$ onto an almost paracontact pseudometric manifold $M$ and let the fibres of $f$ be pseudo-Riemannian submanifolds of $\bar{M}$. If $U, V$ are the non-lightlike unit vertical vector fields and $\bar{X}, \bar{Y}$ are the non-lightlike unit horizontal vector fields, then one has

$$
\begin{gathered}
\bar{B}(U, V)=\bar{B}^{v}(U, V), \\
\bar{B}(\bar{X}, U)=-2\left\|\mathscr{T}_{U} \bar{X}\right\|^{2}, \\
\bar{B}(\bar{X}, \bar{Y})=B(X, Y) \circ f .
\end{gathered}
$$

Proof. Using results of Lemma 19 in (95), we have

$$
\begin{aligned}
\bar{B}(U, V)= & \bar{B}^{v}(U, V)-\bar{g}\left(\bar{J}\left(\mathscr{T}_{U} V\right), \bar{J}\left(\mathscr{T}_{U} V\right)\right) \\
& -\bar{g}\left(\bar{J}^{2}\left(\mathscr{T}_{U} V\right), \mathscr{T}_{U} V\right) \\
= & \bar{B}(U, V)+\bar{g}\left(\mathscr{T}_{U} V, \mathscr{T}_{U} V\right)-\bar{g}\left(\mathscr{T}_{U} V, \mathscr{T}_{U} V\right) \\
= & \bar{B}^{v}(U, V) .
\end{aligned}
$$

Applying results of Lemma 19 in (96), we have

$$
\begin{aligned}
\bar{B}(\bar{X}, U)= & \bar{g}\left(\left(\bar{\nabla}_{U} \mathscr{A}\right)_{\bar{X}}(\bar{J} \bar{X}), \bar{J} U\right)-\bar{g}\left(\left(\bar{\nabla}_{\bar{J} U} \mathscr{A}\right)_{\bar{X}}(\overline{J X}), U\right) \\
& +2\left\|\mathscr{A}_{\bar{X}} U\right\|^{2}-2\left\|\mathscr{T}_{U} \bar{X}\right\|^{2} .
\end{aligned}
$$

Since by Theorem 21 the horizontal distribution is integrable, we have $\mathscr{A}=0$, which implies

$$
\bar{B}(\bar{X}, U)=-2\left\|\mathscr{T}_{U} \bar{X}\right\|^{2} .
$$

In view of $\mathscr{A}=0,(104)$ follows from (97). 
Theorem 32. Let $f: \bar{M}^{m} \rightarrow M^{n}$ be a paracomplex paracontact pseudo-Riemannian submersion from a paraKähler manifold $\bar{M}$ onto an almost paracontact pseudometric manifold $M$ and let the fibres of $f$ be pseudo-Riemannian submanifolds of $\bar{M}$. If $U, \bar{X}$ are non-lightlike unit vertical and non-lightlike unit horizontal vector fields, respectively, then one has

$$
\begin{gathered}
\bar{H}(U)=\bar{H}^{v}(U)-2\left\|\mathscr{T}_{U} U\right\|^{2}, \\
\bar{H}(\bar{X})=H(X) \circ f .
\end{gathered}
$$

Proof. Since $f$ is the paracomplex paracontact pseudoRiemannian submersion from a para-Kähler manifold $\bar{M}$ onto an almost paracontact pseudometric manifold $M$, by (16) and equations of Lemma 19, we have

$$
\begin{aligned}
& \bar{g}\left(\mathscr{T}_{\bar{J} U}(\bar{J} U), \mathscr{T}_{U} U\right)=\bar{g}\left(\bar{J}^{2}\left(\mathscr{T}_{U} U\right), \mathscr{T}_{U} U\right)=\left\|\mathscr{T}_{U} U\right\|^{2}, \\
& \bar{g}\left(\mathscr{T}_{U}(\bar{J} U), \mathscr{T}_{U}(\bar{J} U)\right)=-\bar{g}\left(\mathscr{T}_{U} U, \mathscr{T}_{U} U\right)=-\left\|\mathscr{T}_{U} U\right\|^{2}
\end{aligned}
$$

and by using the above results in (100), we have

$$
\begin{aligned}
\bar{H}(U) & =\bar{H}^{v}(U)-\left\|\mathscr{T}_{U} U\right\|^{2}-\left\|\mathscr{T}_{U} U\right\|^{2} \\
& =\bar{H}^{v}(U)-2\left\|\mathscr{T}_{U} U\right\|^{2} .
\end{aligned}
$$

Again, since horizontal distribution is integrable, we have $\mathscr{A}=0$, and putting it in (101), we obtain (111).

\section{Conflict of Interests}

The authors declare that there is no conflict of interests regarding the publication of this paper.

\section{Acknowledgment}

Uma Shankar Verma is thankful to University Grant Commission, New Delhi, India, for financial support.

\section{References}

[1] B. O'Neill, "The fundamental equations of a submersion," The Michigan Mathematical Journal, vol. 13, pp. 459-469, 1966.

[2] B. O'Neill, Semi-Riemannian Geometry with Applications to Relativity, vol. 103 of Pure and Applied Mathematics, Academic Press, New York, NY, USA, 1983.

[3] A. Gray, "Pseudo-Riemannian almost product manifolds and submersions," vol. 16, pp. 715-737, 1967.

[4] J. P. Bourguignon and H. B. Lawson, A Mathematicians Visit to Kaluza-Klein Theory, Rendiconti del Seminario Matematico, 1989.

[5] S. Ianus and M. Visinescu, "Space-time compactification and Riemannian submersions," in The Mathematical Heritage, G. Rassias and C. F. Gauss, Eds., pp. 358-371, World Scientific, River Edge, NJ, USA, 1991.

[6] J. P. Bourguignon and H. B. Lawson, Jr., "Stability and isolation phenomena for Yang-Mills fields," Communications in Mathematical Physics, vol. 79, no. 2, pp. 189-230, 1981.
[7] B. Watson, "G, G'-Riemannian submersions and nonlinear gauge field equations of general relativity," in Global AnalysisAnalysis on Manifolds, T. Rassias and M. Morse, Eds., vol. 57 of Teubner-Texte zur Mathematik, pp. 324-349, Teubner, Leipzig, Germany, 1983.

[8] C. Altafini, "Redundant robotic chains on Riemannian submersions," IEEE Transactions on Robotics and Automation, vol. 20, no. 2, pp. 335-340, 2004.

[9] M. T. Mustafa, "Applications of harmonic morphisms to gravity," Journal of Mathematical Physics, vol. 41, no. 10, pp. 69186929,2000

[10] B. Watson, "Almost Hermitian submersions," Journal of Differential Geometry, vol. 11, no. 1, pp. 147-165, 1976.

[11] D. Chinea, "Almost contact metric submersions," Rendiconti del Circolo Matematico di Palermo II, vol. 34, no. 1, pp. 319-330, 1984.

[12] D. Chinea, "Transference of structures on almost complex contact metric submersions," Houston Journal of Mathematics, vol. 14, no. 1, pp. 9-22, 1988.

[13] Y. Gündüzalp and B. Sahin, "Para-contact para-complex pseudo-Riemannian submersions," Bulletin of the Malaysian Mathematical Sciences Society.

[14] I. Sato, "On a structure similar to the almost contact structure," Tensor, vol. 30, no. 3, pp. 219-224, 1976.

[15] P. K. Rashevskij, "The scalar field in a stratified space," Trudy Seminara po Vektornomu i Tenzornomu Analizu s ikh Prilozheniyami k Geometrii, Mekhanike i Fizike, vol. 6, pp. 225248, 1948.

[16] P. Libermann, "Sur les structures presque paracomplexes," Comptes Rendus de l'Académie des Sciences I, vol. 234, pp. 25172519, 1952.

[17] E. M. Patterson, "Riemann extensions which have Kähler metrics," Proceedings of the Royal Society of Edinburgh A. Mathematics, vol. 64, pp. 113-126, 1954.

[18] S. Sasaki, "On differentiable manifolds with certain structures which are closely related to almost contact structure I," The Tohoku Mathematical Journal, vol. 12, pp. 459-476, 1960.

[19] S. Zamkovoy, "Canonical connections on paracontact manifolds," Annals of Global Analysis and Geometry, vol. 36, no. 1, pp. 37-60, 2009.

[20] D. E. Blair, Riemannian Geometry of Contact and Symplectic Manifolds, vol. 23 of Progress in Mathematics, Birkhäuser, Boston, Mass, USA, 2002.

[21] V. Cruceanu, P. Fortuny, and P. M. Gadea, "A survey on paracomplex geometry," The Rocky Mountain Journal of Mathematics, vol. 26, no. 1, pp. 83-115, 1996.

[22] P. M. Gadea and J. M. Masque, "Classification of almost paraHermitian manifolds," Rendiconti di Matematica e delle sue Applicazioni VII, vol. 7, no. 11, pp. 377-396, 1991.

[23] M. Falcitelli, S. Ianus, and A. M. Pastore, Riemannian Submersions and Related Topics, World Scientific, River Edge, NJ, USA, 2004.

[24] E. G. Rio and D. N. Kupeli, Semi-Riemannian Maps and Their Applications, Kluwer Academic Publisher, Dordrecht, The Netherlands, 1999.

[25] B. Sahin, "Semi-invariant submersions from almost Hermitian manifolds," Canadian Mathematical Bulletin, vol. 54, no. 3, 2011. 


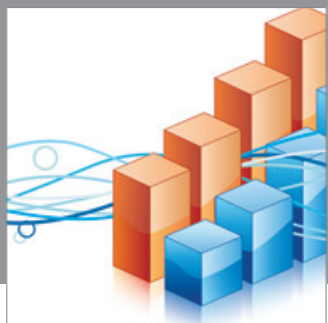

Advances in

Operations Research

mansans

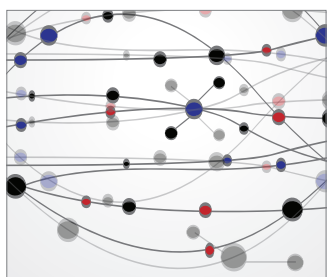

The Scientific World Journal
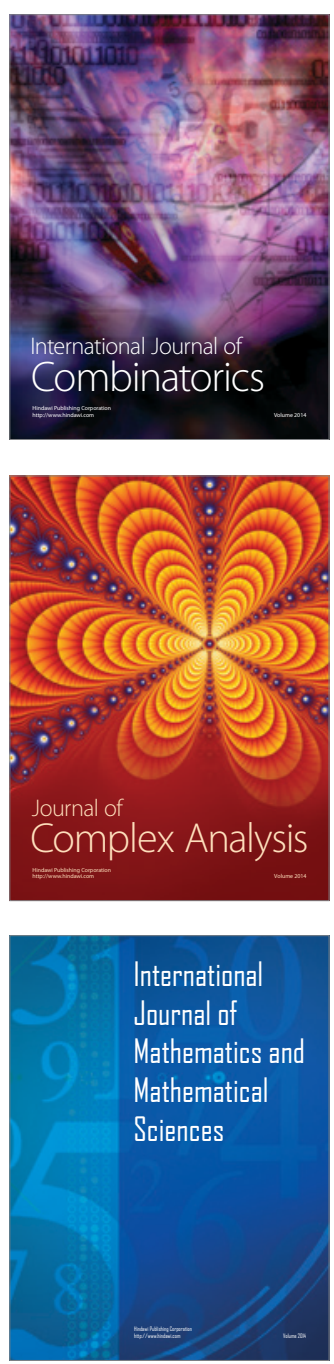
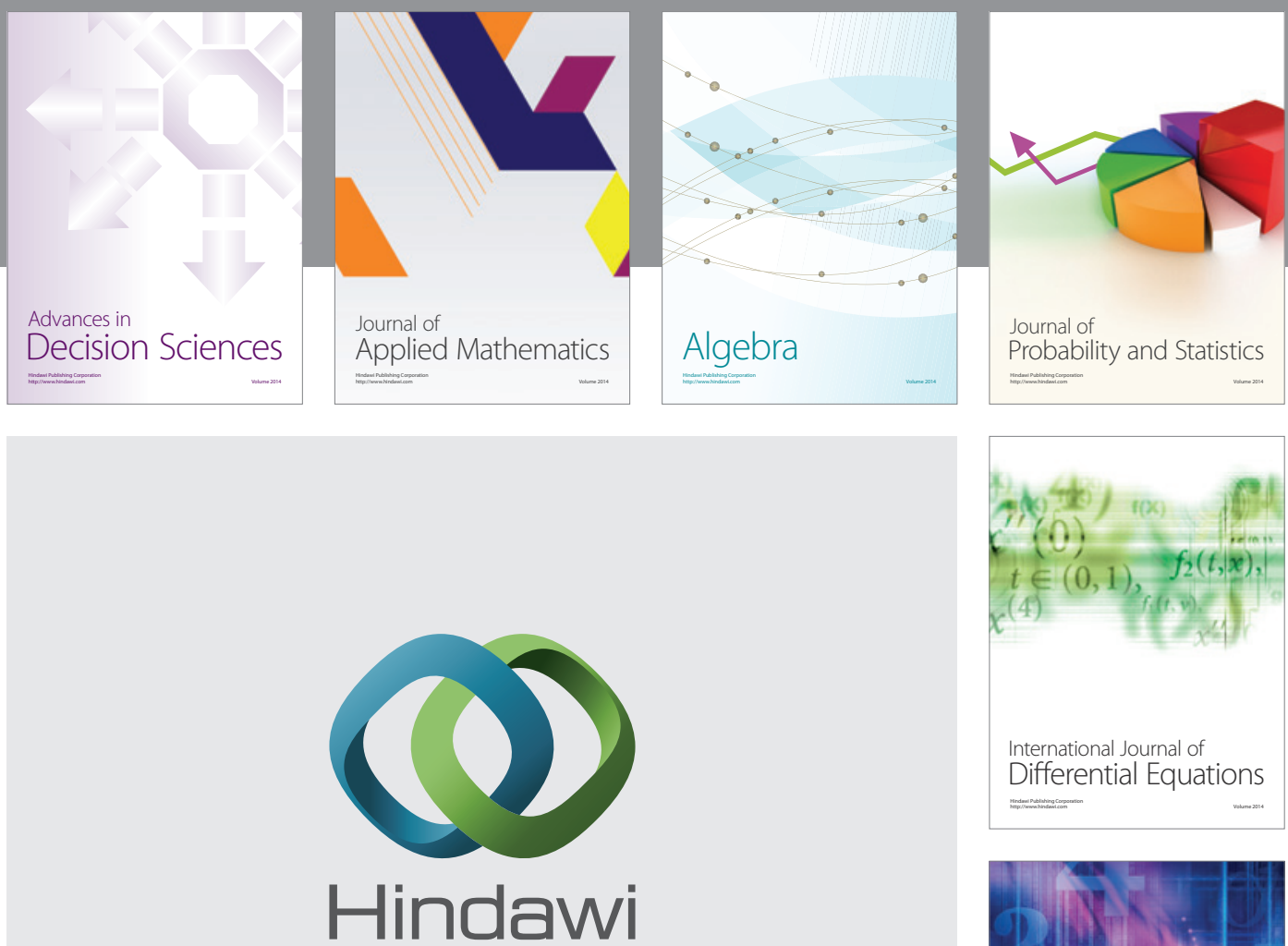

Submit your manuscripts at http://www.hindawi.com
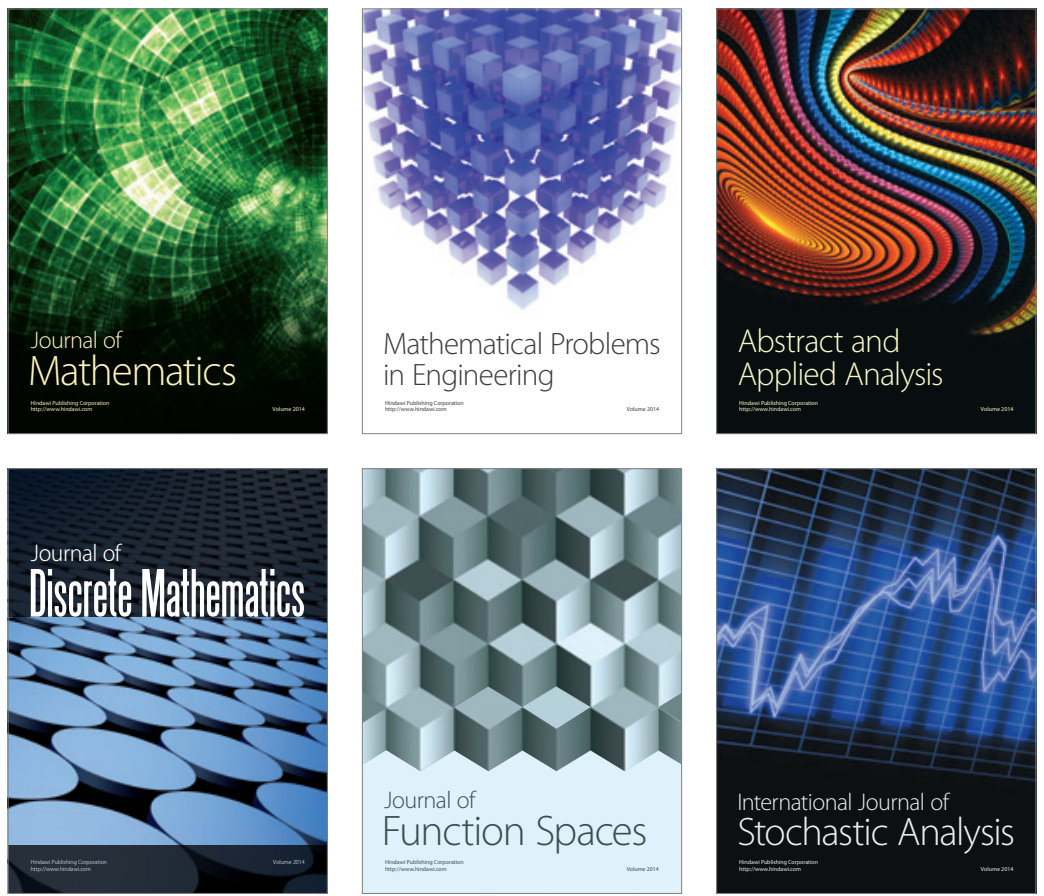

Journal of

Function Spaces

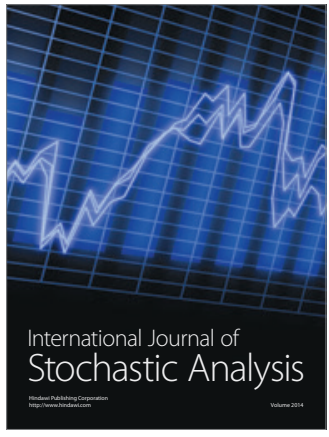

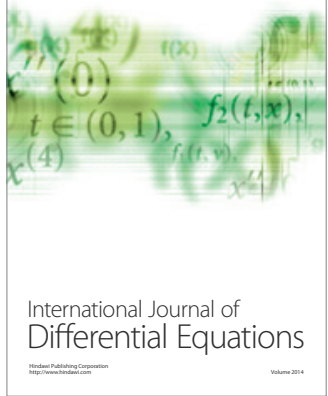
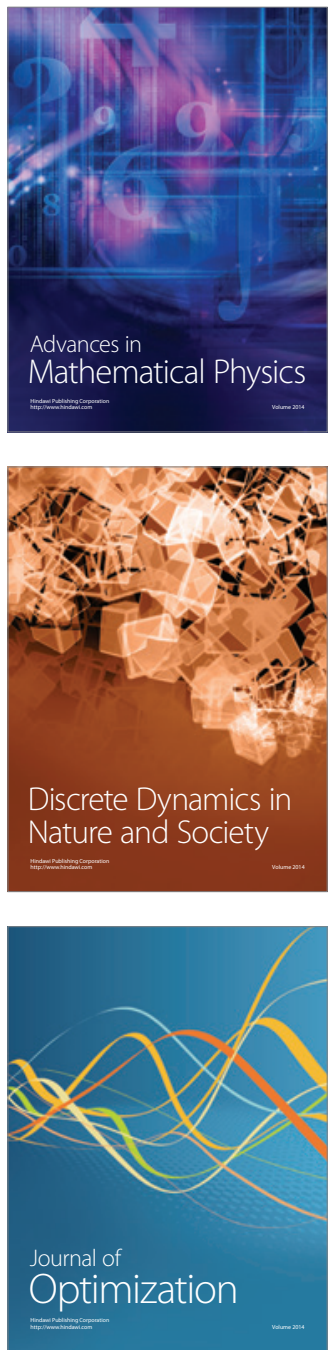